\title{
Operant variability: Evidence, functions, and theory
}

\author{
ALLEN NEURINGER \\ Reed College, Portland, Oregon
}

\begin{abstract}
Although responses are sometimes easy to predict, at other times responding seems highly variable, unpredictable, or even random. The inability to predict is generally attributed to ignorance of controlling variables, but this article is a review of research showing that the highest levels of behavioral variability may result from identifiable reinforcers contingent on such variability. That is, variability is an operant. Discriminative stimuli and reinforcers control it, resulting in low or high variability, depending on the contingencies. Schedule-of-reinforcement effects are orderly, and choosing to vary or repeat is lawfully governed by relative reinforcement frequencies. The operant nature of variability has important implications. For example, learning, exploring, creating, and problem solving may partly depend on it. Abnormal levels of variability, including those found in psychopathologies such as autism, depression, and attention deficit hyperactivity disorder, may be modified through reinforcement. Operant variability may also help to explain some of the unique attributes of voluntary action.
\end{abstract}

Behaving in an unusual, variable, or unpredictable manner is sometimes functional. An individual may sample new paths to reach a goal, invoke varied strategies when competing with an opponent, and generate novel combinations of images and concepts when doing scientific or artistic work. Where does such variability come from? Why can some individuals "be loose," avoid ruts, and engage in novel actions, whereas others seem set in their ways? How do we increase or decrease variability when it is important to do one or the other - to behave in a nontraditionalor creative way when that is needed, and to repeat a practiced, predictable response when situations so demand? One answer to these questions has rarely been examined-namely, that behavioral variability is controlled by its consequences. In other words, variability can be directly reinforced-increased, maintained, or decreased - through the presentation of reinforcers contingent on levels of variability. To state it yet another way, variability is an operant dimension of behavior, molded by its consequences, learned, or emitted voluntarily. Of course, there are other influences on variability, including noise inherent in all physical things and accidents in the environment. However, an understanding of operant variability may help us to explain many fascinating aspects of thought and behavior.

In this article, evidence for operant variability, its relations to other sources of variation, and its functions, explanations, and implications are examined. Some of the

Much of the research from my laboratory was supported by the National Science Foundation. I thank my many Reed College student co-workers for their skill, creativity, persistence, and good humor. I also thank Armando Machado and Seth Roberts for helpful comments, and John Wixted for his gentle persistence in getting me to write this paper. Correspondence concerning this article should be sent to A. Neuringer, Reed College, Portland, OR 97202 (e-mail: allen.neuringer@ reed.edu). topics covered are the following: Variability is controlled by discriminative stimuli and reinforcing consequences, which are characteristics of other operant dimensions. The precise contingencies make a difference in the levels and types of variation that are produced. People and animals choose whether, when, and how much to vary, much as they choose among any other set of operant alternatives. Reinforcement of variations facilitates acquisition of new responses, especially difficult-to-learn ones. Creativity, exploration, and problem solving may depend partly on operant variability. Some psychopathologies, such as attention deficit hyperactivity disorder (ADHD), depression, and autism, may manifest abnormal variability, but reinforcement can produce more normal levels. Reinforced variability may also help to explain the voluntary nature of operant behavior. These and other topics will be explored, but first some terms will be defined.

\section{Definitions}

Operant dimensions are response attributes or parameters that both influence reinforcers and are influenced by them. Examples include response rate, force, duration, location, topography and, as will be seen, variability. Although we often refer to "operant responses," in fact what is generally meant is a combination of dimensions-for example, a press of the left lever of at least a 10 -g force with an interresponse time (IRT) of no more than $1 \mathrm{sec}$. Variability implies dispersion and unpredictability, but it can also refer to a continuum ranging from repetitive at one end to stochastic at the other. Context will indicate the intended meaning. The terms stochastic and random, which will be used interchangeably, imply the highest variability, with the probability of a response being independent of prior responses. Both terms require precisely defined sets of possibilities; for example, a coin 
has two possible states, heads up and tails up. Novel responses are those that have not previously been observed, and the term generally refers to the behavior of a given organism, although sometimes it refers to that of a group or culture. Creative responses are novel or variable ones that meet an additional criterion, often an aesthetic (e.g., artistic) one, but sometimes a functional (e.g., scientific) one. Lastly, many of the experiments to be reviewed compare variable and repeated operants, an example of the latter being repeated responses by a rat on a single lever under the control of reinforcement schedules.

\section{Objections}

Objections are often raised to the suggestion that variability can be reinforced. For example, a reinforcer affects the response that produces it, but variability refers to a set of responses, not a single one. How can a set of events, possibly extending far back in time, be reinforced? Another objection is that reinforcement, by its nature, organizes and strengthens responses, and reinforcement of variability is therefore not possible. Another is that attempting to reinforce variability is inconsistent with two basic goals of behavioral psychologistsnamely, to predict behavior and to control it. Another is that good experimental procedures should minimize variability, not increase it. Yet another is that variability is perceived or posited only when an observer is ignorant of its underlying causes. Finally, adherents of a determinist philosophy may argue that variability does not exist. For these reasons, reinforcement of variability is said to be undesirable, perhaps impossible, and, for some, not meaningful. The evidence, as we will see, indicates otherwise: The study of reinforced variability provides a new way to view operant behavior and opens new areas of research.

\section{INITIAL STUDIES}

\section{Reinforcement of Variable and Novel Responses}

The proposal that variable behaviors can be reinforced goes back at least 40 years, but its history has been a contentious one. In this section, some of that history and the surrounding debate will be described. Maltzman (1960) was among the first to suggest that human subjects could learn to generate low-probability responses, in this case verbal responses to stimulus words. Experimental subjects were asked to devise many different associates to the same word, whereas a control group had to produce only one. Because of their training, the experimental subjects came to generate lower probability associates than did the controls. Although these findings imply that low-probability responding can be trained, there was no clear evidence that reinforcement played a role.

However, reinforcement was clearly involved when Pryor, Haag, and O'Reilly (1969) rewarded porpoises for jumping, turning, or swimming in novel ways. In each session, the trainer waited for a response that had not previously been observed, and that response became the reinforced target throughout the session. The porpoises came to emit novel and complex behaviors whenever they entered the experimental context. Indeed, they behaved in ways never before seen in the species. This was an influential study, and it led to additional research.

Goetz and Baer (1973), for example, rewarded preschool children for block-building constructions that differed from one another. A particular form was reinforced upon its first occurrence within a session, but, in contrast to the procedures used by Pryor et al. (1969), many different forms were reinforced during each session. As training proceeded, the children constructed increasingly different forms, including novel ones. When reinforcers were later contingent upon repetitions, the children repeatedly constructed a single form. Thus, variability and repetition were controlled by consequences.

Although Maltzman (1960), Pryor et al. (1969), and Goetz and Baer (1973) advantageously studied naturally occurring behaviors, some problems were also identified. In Pryor et al., the responses became so complex that the experimenters had difficulty distinguishing among them. In both Pryor et al. and Goetz and Baer, the experimenters had to identify each form or response pattern and decide when a new one had occurred prior to each reinforcement. In all three studies, novel responses became increasingly difficult, since the "easy" ones were emitted first. Also, in the Maltzman and the Goetz and Baer studies, verbal feedback was provided, and the contribution of descriptive information was difficult to distinguish from that of reinforcement. Alternative methods were needed, especially if the goal was to analyze reinforced variability experimentally, and Blough (1966) provided an ingenious one.

\section{Reinforcement of Variable IRTs}

Blough (1966) rewarded pigeons for pecking a response key if the time between consecutive pecks, or IRT, had occurred least frequently in the recent past. This constituted an attempt to see if pigeons could learn to respond randomly over time, similarly to what takes place during the emission of atomic particles at random intervals during radioactive decay. The birds were surprisingly successful. To get a sense of the reinforcement contingencies, imagine a stochastic process in which responses occurred with a 0.5 probability during each second since the previous response. Response probability would be 0.5 during the first second, and, if a response had not occurred, it would be 0.5 during the next second, and so on. Given 1,000 responses, approximately 500 would occur during the first second since the previous response; that is, in the $0-1-\mathrm{sec}$ time frame, or what Blough referred to as a "bin," 250 responses would occur in the 1-2-sec period since the previous response, 125 in the $2-3-$ sec bin, and so on. Blough created 16 IRT bins, adjusting their sizes so that a random generator would produce equal numbers in the counters associated with each bin. Because, as has just been described, many more short than long IRTs are emitted, bin size was small 
at the short end and large at the long end, and increased systematically across the IRT range. For the response to be reinforced, a pigeon's IRT had to fall in the bin that contained the fewest prior entries, across a moving window of 150 responses. Fine-grained analyses of the pigeons' performances showed that successive responses were not truly independent, as would be expected from a random generator-the tendency for long IRTs to follow long ones and for short IRTs to follow short ones was greater than would be expected from a random emitterbut this might have been due to particular aspects of the procedure, including the facts that short IRTs were reinforced somewhat more frequently than longer ones, and that if there were few entries in a bin (because the bird had not been responding at that IRT), then successive repetitions of the same IRT could be reinforced repeatedly. Despite these problems, the data were impressively close to those of a random model, as is shown in the top panel of Figure 1. To the extent that the functions are linear on the semilogarithmic coordinates, the behavior approximates that of a stochastic process. The advantages of Blough's procedure were that response classes were defined objectively and independently of a human observer, reinforcers were administered automatically, thousands of responses were emitted per session, and sessions could continue indefinitely without changes in response difficulty or contingency.

Shimp (1967) attempted to extend Blough's (1966) procedure to pigeon choices. Because choices are currently a major focus of interest, and because there were problems with Shimp's procedure, it will be discussed in some detail. Pigeons pecked two keys, left (L) and right (R), in a continuous stream, such as LLLLRRLLRRRLRLLL; the data were analyzed on line in terms of overlapping sets of four responses. In the example just given, assume that each new response is added to the right of the string. The most recent set was composed of RLLL, the second most recent (moving to the left by one response) of LRLL, the third of RLRL, and so on. Given two alternatives, $L$ and $\mathrm{R}$, and sets consisting of four responses, there are $2^{4}$, or 16, possible sequences: LLLL, LLLR, LLRL . . RRRR. Reinforcement was provided if the most recent response had terminated a sequence that had occurred less frequently than all other 15 possible sequences, over a moving window of 96 responses. Although the resulting choice distributions were quite variable in some respects, the results did not match those of a random model and were difficult to analyze, possibly because of the following problem.

Assume that 16 counters represent the 16 sequences of responses, and that one count is added to each counter whenever its associated sequence is emitted. If LLLL has just been emitted, one count will be added to that counter. Another L will increase the same counter, and each successive $\mathrm{L}$ will add another. If LLRR has just been emitted, however, thereby adding one count to its associated counter, four additional responses (not 1, as in the LLLL case) will be required to add again to the same
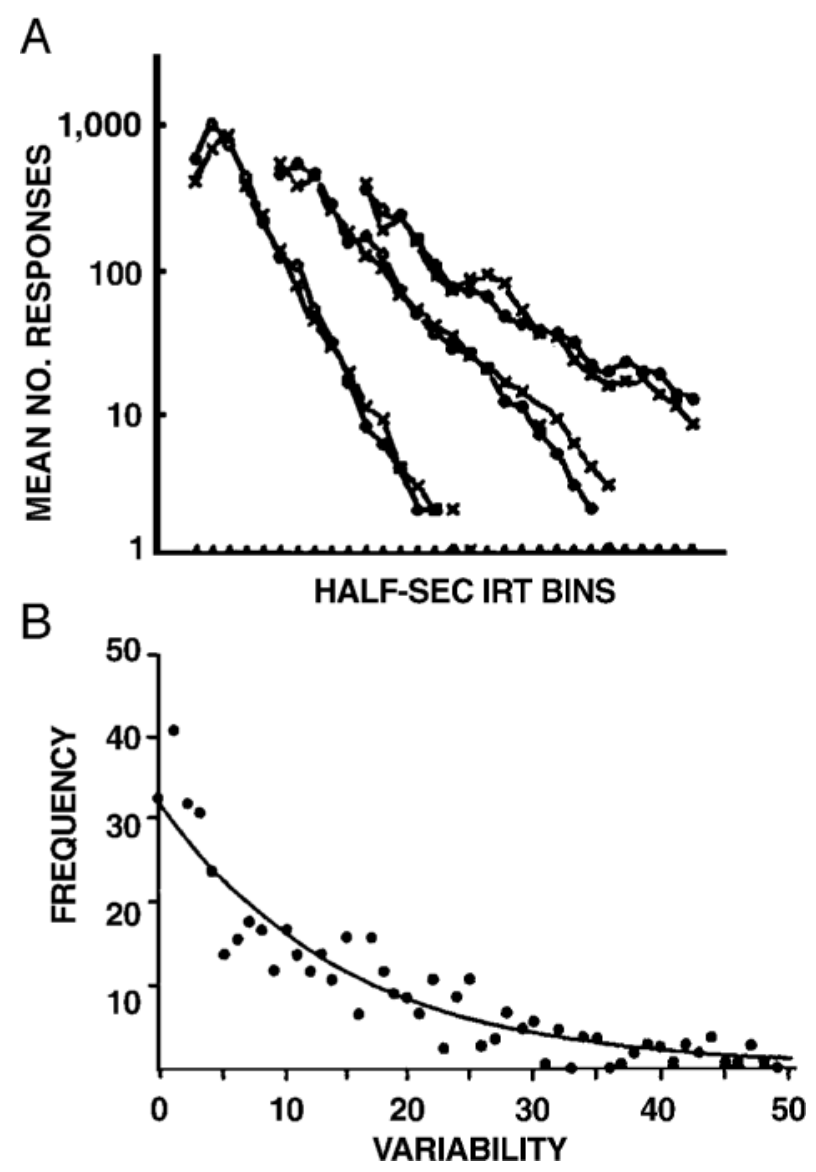

Figure 1. (A) Data from one pigeon in Blough (1966) under different parameter conditions (the three sets of lines) with replication at each parameter. Shown are the mean numbers of responses emitted in each half-second IRT bin, a straight line being expected from a stochastic responder. Adapted from "The reinforcement of least frequent interresponse times," by D.S. Blough, 1966, Journal of the Experimental Analysis of Behavior, 9, p. 587. (B) Data (points) from one pigeon at the end of training in Machado (1989). The line represents the frequencies expected of a random model. From "Operant conditioning of behavioral variability using a percentile reinforcement schedule,' by A. Machado, 1989, Journal of the Experimental Analysis of Behavior, 52, p. 161. Both figures copyrighted by the Society for Experimental Analysis of Behavior. Reprinted with permission.

counter. Therefore, 16 successive responses will be required for four instances of LLRR (namely, LLRRLLRRLLRRLLRR), as opposed to seven for L (LLLLLLL). Although a random process would generate equal frequencies of all sequences, including LLLL and LLRR, the distribution of successive occurrences would differ, thereby affecting the distribution of reinforcements. That is, LLLL and RRRR would tend to be more "clumped" than would LLRR and RRLL, which would be more distributed. Similar considerations apply to all other sequences. That these differences might affect choice distributions is indicated by the many studies showing that the distribution of reinforcements over time, given equal 
average reinforcement frequencies, affects choices; for example, choices are affected differently by variable-interval (VI) than by fixed-interval (FI) schedules of reinforcement.

Wilson (1986) devised one solution to this problem. Wilson's procedure is complex but, in brief, responding was analyzed in a manner that avoided overlapping sequences (which was the cause of the clumping in Shimp's, 1967, procedure). Four sets of counters were created. Using the same example given above, in the first set, one count was added to each counter representing RLLL, RRRL, RRLL, and LLLL, so that successive trials were completely independent. (Note the absence of overlap.) When the next response was emitted, sequences began with that responselet us assume that the next response, added to the right of the series, was an $\mathrm{L}$-and therefore, in the example given, the second set was created by offsetting responses one place to the left. Now one was added to LLLL, with previous counts added to RRLR, RLLR, LLLR, and so on. A similar procedure was followed for each of the remaining two sets. Each response therefore terminated a four-response sequence in one and only one of the four sets, and reinforcement was based on satisfaction of the contingency within that set only. Wilson's procedure had other aspects, but for present purposes it suffices to note that the dependencies in Shimp's procedure were avoided and, when pigeons were tested under these contingencies, they generated choices that more closely approximated those of a random model. Hopefully, this lengthy explanation indicates the importance of precise contingencies when one is attempting to reinforce variability. An advantage of Shimp's and Wilson's procedures was that every response could be reinforced, at least potentially, as was the case for each of the other procedures discussed until now. However, a simpler way to avoid the problems just described is to require a fixed number of responses in each trial, with reinforcement possible only at completion of the trial. As will be seen, most recent studies have used the latter tactic.

The evidence generally supported the claim that behavioral variability could be reinforced, but the agreement about these findings was short lived. Schwartz (1980, 1982) reached the opposite conclusion, and his research will be considered in detail.

\section{Schwartz's Research}

Schwartz $(1980,1982)$ used fixed-length trials consisting of eight responses per trial by pigeons across two response keys. During an initial control phase, a trial terminated with food if it contained exactly four $L$ and four $\mathrm{R}$ responses, regardless of the particular sequence of Ls and Rs. However, if either key was pecked a fifth time, the trial terminated immediately with timeout. A $5 \times 5$ light matrix was provided to assist the birds in this task. At the beginning of a trial, the upper-left corner light was illuminated, and all others were off. An L peck moved the light one column to the right, and an $\mathrm{R}$ peck moved it one row down. Any sequence of four $L$ and four $R$ responses would therefore move the light from upper left to lower right, and the trial would end with food. Each of the birds came to emit a single sequence with high probability, in most cases LLLLRRRR or RRRRLLLL. A relatively low success rate at the end of 40 training sessions indicated that the task was harder than it might have appeared: Only $66 \%$ of trials ended with food, and the remaining $34 \%$ ended with timeout, because, within a given trial, one of the keys had been pecked a fifth time.

Schwartz (1982) then required that the sequence in the current trial differ from that of the just preceding trial. This will be referred to as a lag 1 variability + constraint contingency ("constraint" indicating the required four $\mathrm{Ls}+$ four Rs). If a bird had emitted LLLLRRRR in the previous trial, for example, any other sequence would be reinforced in the next trial, assuming that it contained four Ls and four Rs, but a repetition led to timeout. The birds performed poorly, only $36 \%$ of the trials now ending with food, because a dominant sequence was often repeated or one of the keys was pecked five times. Hypothesizing that this failure to reinforce variability may have been due to the birds' extended experience under the initial control condition (in which variations were not required and any sequence of four Ls and four Rs was reinforced), Schwartz (1982, Experiment 1) again tried the variability-requiring procedure with naive subjects. Again the birds failed. In another experiment, Schwartz (1982, Experiment 2) explicitly trained birds to alternate between LLLLRRRR and RRRRLLLL sequences, and then introduced the lag 1 variability + constraint contingency, but again the birds failed to vary. Schwartz (1982) concluded that variability could not be reinforced, and offered a number of possible explanations for why his results differed from the previous findings: Perhaps Maltzman's (1960) and Goetz and Baer's (1973) findings were due to the information provided-verbal indications from the experimenters that subjects should varyrather than to reinforcement. Perhaps Pryor et al.'s (1969; and by implication, Goetz \& Baer's, 1973) results were due to brief extinction periods in which reinforcers were withheld (because variations were not forthcoming), rather than to direct reinforcement of variability. Perhaps Blough's (1966) results were specific to IRT and not relevant to response forms or choices. These explanations all imply that it is not possible to reinforce variability, or that it is possible only in narrowly defined cases. As will be seen, Schwartz's (1982) results readily met the test of replication, but there was a problem nevertheless with his conclusion.

\section{Schwartz Explained}

Page and Neuringer (1985) simulated Schwartz's (1982) lag 1 variability + constraint procedure with random "responses" generated by a computer. Although the simulation generated $\mathrm{L}$ and $\mathrm{R}$ with equal probabilities $(0.5)$, "reinforcement" was obtained on only $29 \%$ of its trials, which was somewhat lower than the level attained by pigeons. The reason is that, of the 256 possible eight-response sequences, only 72 contained exactly four Rs and four Ls 
and, therefore, the random simulation often failed to meet the $4 \mathrm{~L}+4 \mathrm{R}$ constraint. The implication was that if the birds attempted to respond randomly, they would be reinforced only rarely.

To test whether the penalty for fifth pecks was responsible for the birds' failure to vary, Page and Neuringer (1985) permitted eight responses across L and R keys without the four responses-per-key requirement. If the current sequence of eight responses differed from that in the last trial, a lag 1 variability contingency (without constraint), reinforcement was provided. Now the pigeons succeeded in varying, attaining reinforcement on more than $90 \%$ of the trials, a rate significantly higher than that observed in Schwartz (1982) and close to that of the simulating random responder under the new procedure. Page and Neuringer also replicated Schwartz's (1982) variability + constraint procedure, and again the pigeons failed, just as did Schwartz's. The evidence was clear: Under Schwartz's (1982) procedure, pigeons failed to vary, but penalty for fifth responses was responsible.

One additional aspect of Schwartz's (1982) procedure might also have contributed to low variability. If the birds intermixed a single preferred sequence-one that met the $4 \mathrm{~L}+4 \mathrm{R}$ constraint - with any other, then $50 \%$ of the trials would end with food, this level of intermittent reinforcement being sufficient to maintain responding in many other situations. For example, if a bird alternated between LLLLL (the fifth L producing a timeout) and a dominant LLLLRRRR sequence, the latter would always result in reinforcement. Birds under the variability + constraint contingencies in fact emitted a dominant sequence with high probability, and many of their rewards were obtained following that sequence (because it had been preceded by a different sequence, often one containing five Ls or five Rs). Thus, failure to vary could be explained by the fact that variability was not differentially reinforced, whereas a single, dominant sequence was. Schwartz (1988) later reviewed this new evidence and concluded that pigeons could indeed be reinforced for responding in random-like fashion.

\section{Yoke Control}

One of Schwartz's (1982) hypotheses is worth further consideration: When variability is reinforced, there are periods during which reinforcement is withheld, because responses did not satisfy the contingencies. As will be discussed below, many studies have shown that extinction elicits variability. Might short-term extinction periods, when reinforcers were not forthcoming, have elicited the variability attributed to reinforcement?

Page and Neuringer (1985) compared two conditions. In one, referred to as Var, reinforcement depended on sequence variations similar to the lag 1 variability contingencies described above. (The matrix of lights was no longer used because it was found not to contribute to response variability.) In the other condition, referred to as Yoke, exactly the same frequency and distribution of reinforcements were provided, but independently of se- quence variations. This was accomplished by having the computer maintain a record of which trials were reinforced and which led to timeout in the Var phase, and using this record to program reinforcers during Yoke. For a particular bird, for example, the first as well as the second trials in a Var session might have been reinforced, but in the third trial the bird may have repeated a sequence, causing a timeout, and so forth. In the Yoke phase, the first and second trials would be reinforced, but the third trial would lead to timeout, whether the bird's responses varied or not. Each bird's own sequence of reinforcements and timeouts in Var was therefore replicated in Yoke. If short-term extinction, or withholding of reinforcement, was responsible, then variability should be equal in the Var and in the Yoke phases. That was not the result: Variability was significantly higher in Var than in Yoke, a finding that has been confirmed many times in our laboratory and in those of others (e.g., Barba \& Hunziker, 2002; Machado, 1992). We conclude that variability was in fact reinforced.

\section{VARIABILITY IS AN OPERANT}

Control by reinforcement is the most notable characteristic of an operant response, but there are others, including precise influence of contingencies, effects of reinforcement frequencies, control by discriminative stimuli, lawful distribution of choices, and extinction. Research in each of these areas supports the hypothesis that behavioral variability is an operant dimension, similar to other operant dimensions. An organism can be reinforced not only for repeating a particular behavior, but for distributing responses across a wide array of possibilities in a way that may be quite unpredictable. The evidence will be reviewed.

\section{Contingencies and Frequencies of Reinforcement}

Contingencies specify what must be done to earn reinforcement. When applied to variability, contingencies specify a set of responses and, in most studies to date, a minimal level of variability within that set. That control by contingencies does not depend on reinforcement frequency was demonstrated by Blough (1966) for pigeon IRTs and by Machado $(1989,1992)$ for pigeon response sequences across $\mathrm{L}$ and $\mathrm{R}$ keys. In both cases, when reinforcement depended on high variability, response variability was high; when intermediate variability was required, the level generated was intermediate; and when lower levels of variability were reinforced, variability was relatively low. Reinforcement frequencies were held constant in these studies and therefore were not responsible for the changes in the levels.

With respect to other operant dimensions, such as response rate, reinforcement frequency often has complex and sometimes inconsistent effects: As reinforcement frequency increases, response rates sometimes increase, sometimes decrease, and sometimes are unchanged. Variability is also affected in seemingly inconsistent ways. 
Some studies report that variability increases when reinforcement frequencies decrease (Boren, Moerschbaecher, \& Whyte, 1978; Tatham, Wanchisen, \& Hineline, 1993; Tremont, 1984), but others report small or no effects (Blough, 1966; Eckerman \& Lanson, 1969; Herrnstein, 1961; Machado, 1989).

Grunow and Neuringer (2002, Experiment 1) independently manipulated variability contingencies and reinforcement frequencies, and their results may help to explain the previous inconsistencies. Four groups of rats were studied, with the contingencies for each group requiring a different minimum level of variability. (A threshold contingency was employed, the details of which will be described below.) In the initial phase, reinforcement was provided whenever the respective contingencies were met (reinforcement frequency was relatively high in this phase) and, as can be expected from the Blough (1966) and Machado (1989) studies described above, levels of variability were directly controlled by the contingencies. In a second phase, with the same variability contingencies held constant, reinforcement was limited to no more than once per minute (VI 1, or intermediate frequency). In a third phase, meeting of the variability contingency was reinforced on an average of only once every 5 min (VI 5 , or low frequency). We observed a significant interaction between reinforcement frequency and variability contingency (Figure 2), which helps to explain some of the previous inconsistencies. In the group that was reinforced for low variability, represented by the triangles in Figure 2, as reinforcement frequency decreased, variability increased slightly; in the group reinforced for high variability, represented by the squares, when reinforcement decreased, variability decreased; and in the intermediate groups, variability was unchanged. Although these effects were small, they reached statistical significance. As can be seen in Figure 2, variability contingencies affected variability more than did reinforcement frequencies, and effects of the latter were influenced by the contingencies.

An example might help to illustrate these complex findings. When low variability is required, perhaps as in assembly-line work, low variability emerges. When high variability is required, perhaps as in artistic work, response variability is high. Now, what happens if the normal rate of reinforcement for each of these tasks is lowered? According to Grunow and Neuringer's (2002) findings, the assembly-line worker's variability would increase, but only slightly, and the artist's variability would de-

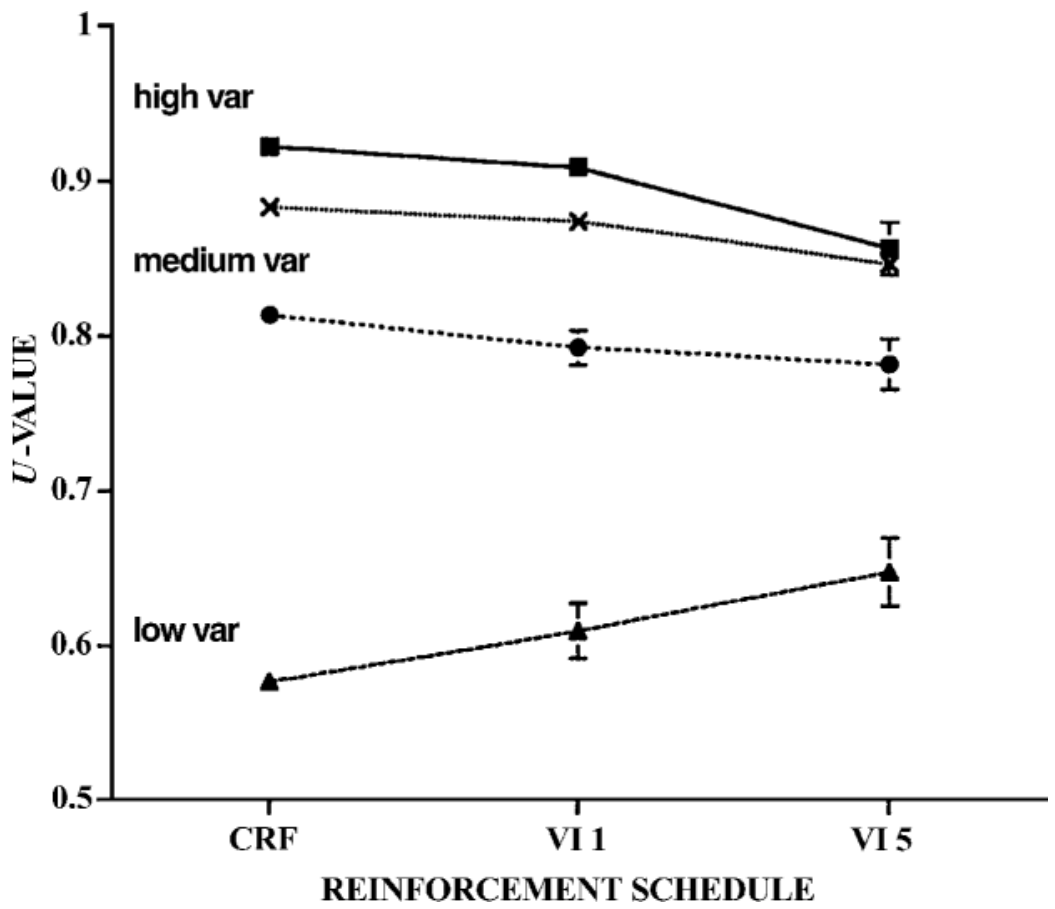

Figure 2. Average $U$-values across groups of rats in Grunow and Neuringer (2002). The highest function (squares) is from a group reinforced for highest sequence variability; the lowest function (triangles) is from a group reinforced under a lenient contingency - that is, low variability sufficed for reinforcement; the other groups (Xs and circles) were reinforced for intermediate levels of variability. Each group was reinforced at three frequencies: each time the contingency was satisfied (continuous reinforcement; i.e., CRF), on an average of not more than once per minute (VI 1), and on an average of not more than once every $5 \mathrm{~min}$ (VI 5). High $U$-values indicate high levels of sequence variability, and low $U$-values indicate relatively repetitive responding. Error bars represent standard errors of the mean. 
crease somewhat. Importantly, the artist would continue to behave much more variably than the assembly-line worker. Both contingency and frequency affect response variability, but contingency appears to be more influential, at least over the range of values studied to date. These findings, if general, are important for those who withhold reinforcement in order to encourage response variations, as is often the case when behavior modifiers attempt to shape new behaviors. Effects of withholding may depend on baseline contingencies, and a more productive procedure might be to reinforce variability directly. We will consider this suggestion further below.

\section{Discriminative Control}

The operant response, as defined by Skinner, is controlled by discriminative stimuli as well as by consequences. For example, red traffic lights are the discriminative stimulus for depressing the automobile brake; the distance of the golf ball from the hole provides a discriminative stimulus for how hard to hit the ball. In the laboratory, discriminative control is often studied with multiple schedules, in which one reinforcement contingency, such as fixed ratio, is operative in the presence of one stimulus, such as a red keylight, and a different contingency-for example, extinction-is operative in a second stimulusfor example, a green keylight. Discriminative control is shown by differences in responding. Variability is also controlled by discriminative cues.

Exteroceptive discriminative stimulus control. Page and Neuringer (1985, Experiment 6) reinforced pigeons for repeating a single sequence, LRRLL, in the presence of blue keylights, and for variable sequences in the presence of red. Blue and red alternated after every 10 reinforcements. The birds learned to repeat in the presence of blue and to vary in the presence of red, and when the stimulus relationships were reversed, the birds reversed their patterns. Cohen, Neuringer, and Rhodes (1990) obtained similar results with rats. Denney and Neuringer (1998) extended this research to show that discriminative control did not depend on reinforced repetitions in one of the components. Again, within the context of a multiple schedule, rats were rewarded for variable sequences under one stimulus (Var) and independently of variability under a second stimulus (Yoke). Frequencies of reinforcement were identical in the two stimulus conditions. That the stimuli exerted significant control over variability was indicated by three main findings. Variability was higher in Var than in Yoke; differences in variability were greatest immediately following stimulus change; and when the discriminative stimuli were removed and all other aspects of the program kept constant, variability immediately converged on an intermediate and identical level in the two components. The conclusion is that operant variability is controlled by discriminative stimuli. These findings are important in terms of both explaining observed levels of variability and controlling them. For tasks in which response variations are important, such as when new operants are being shaped or when an individual is attempting to behave creatively or solve problems, cues that indicate "Vary!" can be helpful.

\section{Discriminative control without exteroceptive stim-}

uli. The relationship between a response and a reinforcer can itself provide a discriminative stimulus, even when external cues, such as lights or tones, are unavailable. For example, under an FI schedule, there is a period during which reinforcers cannot be obtained. The subject learns to pause following each reinforcement, although no cues other than the reinforcer are provided. Another case is the mixed schedule, identical to the multiple schedule described above, but with no external cues. Animals sometimes learn to respond differentially to the consequences of their behaviors, even under such stimulus-impoverished conditions (Ferster \& Skinner, 1957). The question studied next was whether response-reinforcer relationships can provide discriminative cues sufficient to control levels of variability. This is an important question because, as was indicated above, it has been assumed that withholding reinforcement elicits variability. The present hypothesis is that absence of reinforcement can sometimes serve as a discriminative cue for operant variability. The distinction between elicited (respondent) and reinforced (operant) variability will be revisited shortly.

Hopson, Burt, and Neuringer (2002) rewarded rats under a mixed schedule in which two periods of time, or components, Var and Repetition (Rep), alternated without any external cues. In the Var component, four-response sequences of $L$ and $R$ leverpresses were reinforced if they met a threshold variability contingency; in the Rep component, only repetitions of LLLL were reinforced. (Probabilities of reinforcement were equalized in the two components by intermittent reinforcement of LLLL in Rep.) The results demonstrated discriminative control despite the absence of exteroceptive cues (see Figure 3). With trials since the unmarked switch into the Var component, shown in the left column, variability increased and LLLL sequences decreased; with trials since the unmarked switch into Rep, shown in the right column, responding became increasingly repetitive until LLLL sequences predominated. The two functions were similar, which is an important finding, in that it indicates discriminative control rather than elicitation. If variability had been elicited because reinforcement was temporarily withheld (when the contingencies were switched), then variability should have increased following entry into both Rep and Var components, but that was not the result.

Therefore, variability that is observed when reinforcement is withheld for short periods, as when a new response is being shaped, may be partly discriminatively controlled despite the absence of external cues; that is, animals and people may learn when it is functional to vary, and some of the cues may come from responseoutcome relationships. Control over this operant variability may be more precise than that elicited by extinction, because, as was noted above, operant contingencies define both the set of acceptable variations and the level 


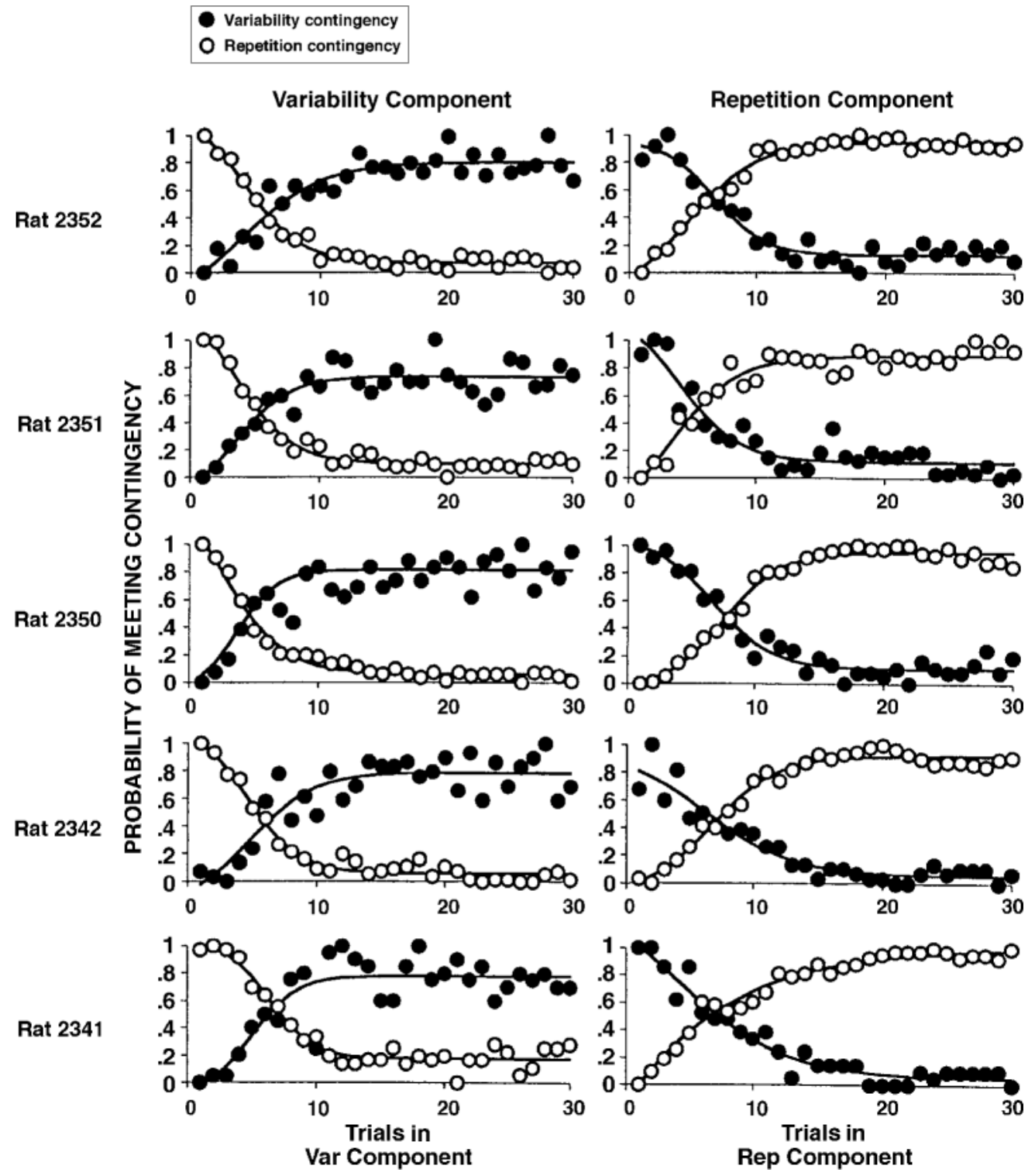

Figure 3. The left column shows the probabilities ( $y$-axis) that each of five rats met a variability contingency (solid circles) versus a repetition contingency (open circles) when they were being reinforced for varying (Hopson, Burt, \& Neuringer, 2002). Along the $x$-axis are trials since the mixed schedule (no cues provided to indicate the component) had switched in to the variability-reinforcing component. The right column shows the corresponding data for performances when reinforcement was contingent upon repetitions, the $\boldsymbol{x}$-axis here indicating trials since the unmarked switch into the repetition-reinforcing component.

of required variability. It may also be maintained much longer because, as will be seen shortly, extinction weakens responding.

\section{Choice}

The frequency of one response relative to others, or response probability, is used as a measure of choice or preference. Choices are found to match the probabilities of their consequences. For example, relative frequencies of pecking one of two keys approximate relative fre- quencies of reinforcement. Neuringer (1992) showed that the same matching relationship also governs choices by pigeons to vary or repeat: The more variations were reinforced, the more the pigeons chose to vary, and similarly for repetitions. Each trial consisted of four $\mathrm{L}$ and $\mathrm{R}$ responses, and reinforcements for varying and for repeating were systematically manipulated. The Var contingency was met whenever the current sequence differed from each of the previous three sequences-a lag 3 criterion. The Rep contingency was satisfied if the cur- 
rent sequence repeated at least one of the previous three. On an average of once every $30 \mathrm{sec}$ (VI $30 \mathrm{sec}$ ), the computer probabilistically primed reinforcement for Var or Rep, the prime being maintained until the reinforcement had been collected. Thus, if the computer primed Var, reinforcement was delivered whenever the lag 3 variability contingency was next met. Similarly, if Rep had been primed, the next Rep sequence was reinforced. Probabilities of Var and Rep reinforcements were systematically manipulated, the two always summing to 1.0 , and a matching-type relationship was obtained (Figure 4, panel A). The relationship was more complex than simple matching, possibly because, if the pigeons chose to vary by responding stochastically, there was some probability, due to chance, that the current sequence would repeat a previous sequence. This was shown by a comparison of pigeon performances to the performance of a computer-simulated stochastic model programmed to match its Var choices to the probability of Var reinforcements: The pigeons' choice distributions were found to be the same as the model's (Figure 4, panel B). Thus, choices to vary or repeat were governed by relative frequencies of reinforcement. An animal can choose whether, when, and how much to vary, a result consistent with those described above from Blough (1966), Machado (1989), and Grunow and Neuringer (2002). Choices of whether or not to vary appear to be governed similarly to all other operant choices.

These findings may have important applications. When an individual repeatedly responds in a way that is nonfunctional, reinforcement of choices to vary may help the individual to emerge from the ineffective pattern. This can be true for those experiencing everyday problems but feeling helpless to change, and it may be especially important for those experiencing depression or who respond in otherwise abnormally stereotyped manners, such as those with autism. These topics will be considered in a later section.

\section{Extinction}

As was indicated above, extinction is often found to increase variability, but almost all studies of this effect involve responses that had previously been repetitive. Antonitis (1951), for example, reinforced rats for poking their noses any where along a $50-\mathrm{cm}$ horizontal opening. Although the exact location of pokes did not matter, with continued training, responses became limited to one or a few locations along the strip. When reinforcement was withheld, variability of location increased (see also Eckerman \& Lanson, 1969). Variability produced by extinction has been reported for many other response dimensions as well, including force (Notterman \& Mintz, 1965), number (Mechner, 1958), topography (Stokes, 1995), and sequence (Balsam, Paterniti, Zechowy, \& Stokes, 2002; Mechner, Hyten, Field, \& Madden, 1997).

The issue is complicated, however, by other studies that show that previously reinforced responses and response patterns are maintained intact during and after extinction. That is, extinction does not break down
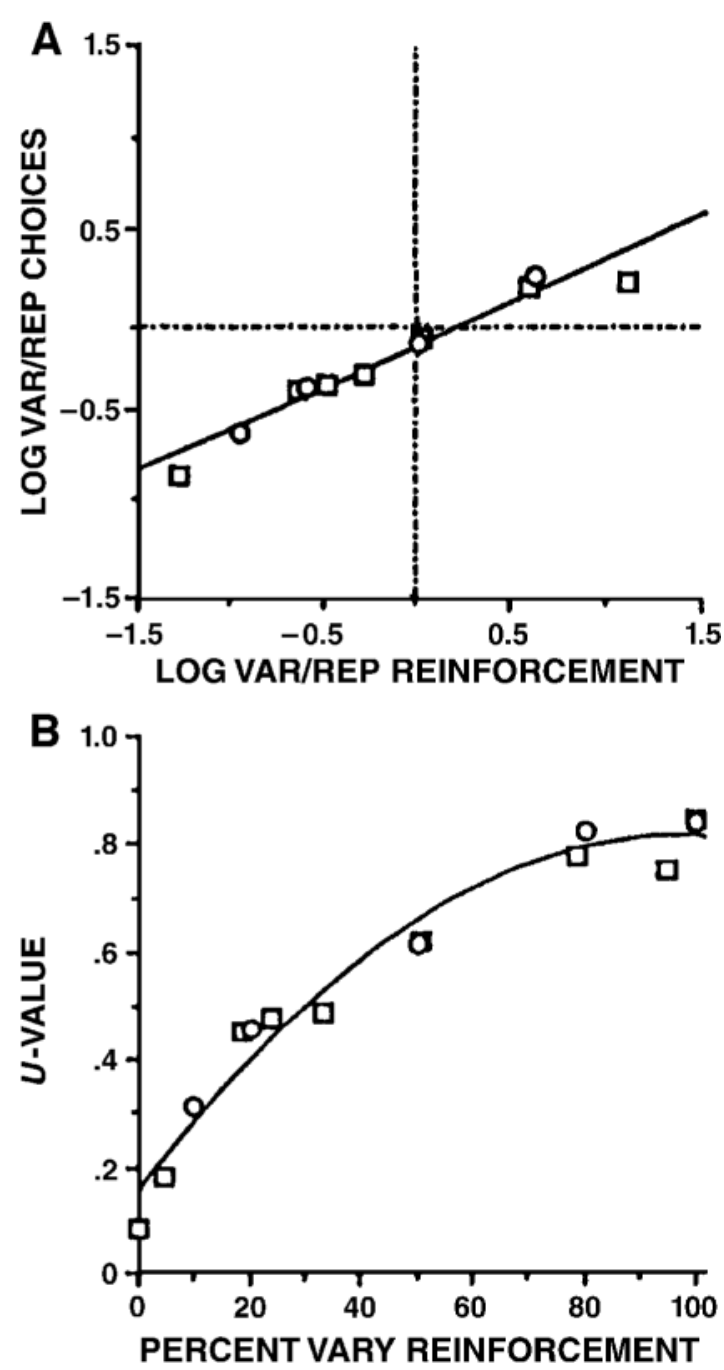

Figure 4. Average performance across a group of pigeons in Neuringer's (1992) study of choices to vary or repeat. (A) Logarithms of the ratio of number of times the pigeons met the Var contingency divided by the times they met the Rep contingency as a function of the analogous numbers of reinforcements received for each. Points represent group averages in two separate experiments. Also shown is the least squares best-fitting line. (B) Changes in $U$-value, an index of response variability, as a function of the obtained percentage of reinforcements for varying. The solid line in panel $B$ represents the performance of a computer-based model that matched its choices to vary (vs. repeat) to its obtained relative frequency of reinforcem ent for varying. From "Choosing to vary and repeat," by A. Neuringer, 1992, Psychological Science, 3, p. 248. Copyright 1992 by the American Psychological Society. Reprinted with permission.

learned patterns. For example, when Schwartz (1981) reinforced pigeons for any sequence of four $\mathrm{L}$ and four $\mathrm{R}$ responses, each bird developed a dominant sequence, as described above, and these sequences were maintained during extinction. Despite decreasing response rates during extinction, response patterns were retained. Similar maintenance of response structure has been observed during extinction following reinforcement of 
choices (Myerson \& Hale, 1988), responding under fixed-interval contingencies (Machado \& Cevik, 1998), visual discriminations (Nevin, 1967), and matching to sample (Cumming, Berryman, Cohen, \& Lanson, 1967). How can stability during extinction be reconciled with increased variability?

Neuringer, Kornell, and Olufs (2001) wrestled with this question in a study of extinction following reinforcement under three different contingencies. An operant chamber contained two levers, left (L) and right (R), on either side of a reinforcement dispenser, and a key (K) in the rear of the chamber, distant from the dispenser. One group of rats, Rep, was reinforced for repeating a fixed sequence of responses, LKR. A second group, Var, was reinforced for variations across the three operanda under a threshold contingency. Reinforcement for a third group, Yoke, was matched to those of the Var animals and was therefore independent of the particular sequences. Because of the unusual configuration of operanda, skewed distributions of sequence probabilities were observed in all of the groups, an outcome that was anticipated and desired in order to document possible increases in variability during extinction-a flattening of the distribution-as well as possible decreases. That is, during the reinforcement phase, the Rep group came to repeat the LKR sequence with high probability; the Yoke group tended to emit the easiest sequences, such as LLL and RRR, but distributed their responses over more sequences than the Rep group did; and the Var group distributed most widely, although, because of differences in the operanda and in their locations, some sequences were more preferred than others, and in particular, sequences ending with a $\mathrm{K}$ response were least likely (the key being farthest from the reinforcement dispenser). Thus, the contingencies resulted in three different distributions, or hierarchies, of sequence probabilities: very steep for Rep, intermediate for Yoke, and flattest for Var.

The main finding was that withholding reinforcement had similar effects in the three groups. In each, response rates fell to low levels across the four sessions of extinction. Furthermore, sequences that were most frequent during the reinforcement phase continued to be most frequent throughout extinction. A slight flattening of the distributions was observed, however, with the lowest probability sequences increasing relative to the reinforcement phase. Thus, because the overall hierarchy of sequence frequencies was maintained, the results indicated stability; but because the distributions were somewhat flattened, the results also showed slightly increased variability. It is as if the strategy during extinction were to keep doing what had worked in the past, but occasionally to do something very different. This complicated story integrates two effects of extinction: increased variability and maintained stability. Most importantly for the present discussion, extinction had similar effects on variable, yoked, and repetitive operants.

One additional comparison is of interest. Recall that Grunow and Neuringer (2002) found that when contin- gencies demanded high variability, obtained levels of variability decreased slightly as reinforcement frequencies were lowered. This result is opposite to the Var group's slight increase in variability just described under extinction conditions. The reason may be that extinctioninduced variability is short lived, lasting only as long as responding continues, perhaps for hours or a few sessions at most, whereas the variability engendered by the VI 5 intermittent schedule of reinforcement in Grunow and Neuringer can be maintained indefinitely. The decrease in Grunow and Neuringer's operant variability may be due to the weakening of the reinforcement effect (i.e., reinforcement is less frequent), whereas the increase following extinction may be a short-term elicited effect. This distinction between reinforced and elicited variability is important and will be discussed further in the next section.

\section{Operant and Respondent Interactions}

Elicited, or what is sometimes referred to as respondent, effects occur when environmental changes that affect behavior do not depend on (are not caused by) the behavior, Pavlovian conditioning being the most commonly studied example. Operant effects are observed when a reinforcer depends on the response to produce it. Although, for historical reasons, respondent and operant effects are often studied separately, most behaviors result from a combination, or interaction, of the two. Variability is no different, and we turn to an example of such an interaction.

As reinforcement is approached under FI or fixedratio (FR) schedules, response rates are commonly observed to increase. Similarly, when reinforcement depends on accuracy, as in discrimination learning or matching to sample, it too is found to increase within the FI or the FR (Nelson, 1978; Nevin, 1967). Cherot, Jones, and Neuringer (1996) asked whether the same pattern held for reinforced variability: Does operant variability increase as reinforcement for varying is approached? The question may be related to such real-world cases as when a composer is rushing to complete a composition before a scheduled performance, or when a programmer is trying to solve a problem before an upcoming meeting. These activities involve, at least to some extent, nonrepetitive, original, or variable thought and behavior. The question is whether variability increases with proximity to, or anticipation of, reinforcement for varying.

Cherot et al. (1996) employed trials consisting of four responses across $\mathrm{L}$ and $\mathrm{R}$ operanda and a lag 4 variability contingency, with rats in one experiment and pigeons in another. Unlike in any of the cases described so far, however, the lag-variability contingency had to be satisfied four times in order for a single reinforcer to be obtained. With "*" indicating successful completion of the Var contingency, "-" indicating failure to meet that contingency, and PELLET indicating the food reinforcer, an example follows: LRLL*; RRRL*; LRLL-; LRLL-; RLLR*; RRRL-; LRRR*+PELLET. Thus, food reinforcement was provided only after the subject had satisfied 
the variability contingency four times, FR 4 . The results were that, as the food reinforcer was approached (i.e., as progress was made through the ratio), the subjects were less and less successful at varying. The probability of meeting the variability contingency in the first trial after reinforcement was higher than it was in the second, which in turn was higher than that in the third trial, and so on. For comparison, a Rep group was reinforced for repeating sequences, once again with reinforcement only after four successful repetitions. Probability of repeating increased with proximity to reinforcement, a finding consistent with the literature but opposite that for the variability group. Thus, approach to reinforcement facilitated operant repetition but interfered with operant variability. Similar variability-interfering effects have been documented for within-trials responses: The probability that a response repeats the just prior response increases within a trial, even when sequence variability is reinforced (McElroy $\&$ Neuringer, 1990; Neuringer, 1991). To return to the example of a composer rushing to complete her composition, she may indeed have increasing difficulty responding creatively under the pressure of a deadline. On the other hand, the soloist may find it easier to repeat the correct performance during practice periods as the concert nears. How can these results be reconciled with the many findings that variability can be reinforced?

An additional result of Cherot et al. (1996) provides an answer. The Var animals responded much more variably overall than did the Rep animals. Although variability in the Var groups decreased with approach to the reinforcer, overall variability was always much higher when it was reinforced (Var group) than when it was not (Rep group). Reinforcement therefore exerts two influences: Overall variability is elevated, sometimes to the highest levels, when it is reinforced; but approach to (and possibly focus on) the reinforcers constrains, or lowers, variability. Similar reinforcement-produced interference effects had been reported previously; for example, when raccoons are reinforced for dropping a "coin" into a slot, the reinforcement results in the animals' holding and rubbing the coin rather than dropping it (Breland \& Breland, 1961). The overall enhancement of variability was of much greater magnitude in Cherot et al. than the decrease with approach was, although both effects were statistically significant. (The notes created by the composer under pressure of deadline will be less readily predicted than will those played by the performer practicing a well-known piece.) This study highlights the importance of interactions between operant effects of reinforcement and elicited effects produced by anticipation of reinforcement, and the results may be relevant to the debate concerning possible detrimental effects of reinforcement on creative behaviors (see the discussion below and Neuringer, in press).

\section{Summary}

Reinforcement controls behavioral variability, as it does other operant dimensions. Levels of variability are precisely governed by schedule contingencies and dis- criminative stimuli. Choices to vary or repeat match relative frequencies of reinforcement. Extinction increases variability slightly, whereas the hierarchies of sequence probabilities are retained, and operant and respondent effects interact. The evidence shows that behavioral variability is an operant, controlled by its consequences.

\section{GENERALITY AND PARAMETERS}

Many different methods, measures, species, and responses have been used in the study of operant variability, and this diversity supports the generality of the phenomenon. As is the case for repeated operants, however, there are parametric constraints. This section briefly reviews the generality and some of the parameters studied to date.

\section{Generality}

Methods. Throughout this section, "response" will signify a response unit, including both an individual response and a multiresponse sequence. Each of the methods to be described increases or maintains variability by reinforcing it.

Novel response procedures reinforce responses upon their first occurrence "ever" (Pryor et al., 1969) or upon their first occurrence within a session (Goetz \& Baer, 1973). Radial-arm maze procedures also reinforce initial (within a given session) entries into arms of a maze and result in a distribution of choices across the available options, the sequence or pattern of choices often being quite variable.

Lag procedures reinforce a response if it has not occurred across a given number of previous trials (Page \& Neuringer, 1985). For example, under lag 5, the current sequence is reinforced only if it has not occurred during any of the previous five trials. A variant is the recurrencetime procedure, which specifies a minimum number of intervening trials before a previously emitted response can again be reinforced. Machado (1989) combined recurrence time with a percentile-reinforcement contingency to generate high levels of variability (see also Machado, 1992). Percentile-reinforcement contingencies base the criterion for reinforcement on the subject's own performance over a previous set of responses (see Galbicka, 1994). For example, a sequence will be reinforced only if it has not occurred over the previous $n$ trials, the value of $n$ continually changing so that $50 \%$ of the trials are reinforced.

Least-frequent response procedures (Blough, 1966; Schoenfeld, Harris, \& Farmer, 1966; Shimp, 1967) reinforce responses that are currently least frequent across a moving window.

Threshold procedures reinforce responses whose relative frequencies fall below a given value. Because this type of procedure was used in the research described above and in many other, more recent studies, details will be provided. In Denney and Neuringer (1998), which will serve as an example, trials consisted of four responses by rats across $L$ and $R$ levers. A running tally was kept of the 
number of occurrences of each of the 16 possible sequences LLLL, LLLR, LLRL, and so on. If the relative frequency of the current sequence-the number of its occurrences divided by the sum of all 16 sequences-was below a specified threshold value, in this case .05 , the rat was rewarded. Recently emitted sequences contributed more to the maintained tally than did those in the distant past because, after each reinforcement, all 16 counters were multiplied by a weighting coefficient, in this case .95. Therefore, the influence of a particular trial decreased exponentially with successive reinforcements. Threshold procedures differ from Blough's (1966) least-frequent procedure in that more than one sequence (or response) can be reinforced at any given time-namely, all those sequences that have relative frequencies below the threshold value. The procedure differs from lag in permitting a given sequence to be reinforced many times in succession, if the associated relative frequency is sufficiently low.

The frequency dependence procedure (Machado, 1992, 1993) is a variant of threshold in which the probability of reinforcement is a continuous function of relative response frequency: The more frequent a response is, the lower the probability is that it will be reinforced.

Statistical feedback procedures provide subjects with comparisons between their performances and that of a model. Neuringer (1986) showed human subjects feedback from 10 statistical tests, thereby enabling them to compare their own distributions of responses to that of a random model. Neuringer and Voss (1993) provided feedback based on a chaotic model. The goal in each of these cases was for the subjects to learn to approximate the model, and the results of these experiments will be discussed below.

Differential reinforcement of switches versus repetitions balance a subject's tendency to engage in each of these patterns. Rather than reinforcement for infrequent responses or random-like distributions, reinforcement is provided sometimes for repeating a response and other times for switching from one response to another. In the twooperandum case, a switch is L followed by R, or R by L; a repetition is $L$ then $L$, or $R$ then $R$. Bryant and Church (1974) found that stochastic-like responding was generated by rats when switches were reinforced with a .75 probability and repetitions with a .25 probability; that is, the rats had a natural tendency to repeat, at least in the environment of this study. Machado (1997) reinforced pigeons for eight pecks across two keys if the sequence of pecks contained at least one (one group of birds) or two (another group) switches. Although the birds could have satisfied these contingencies by repeating a single sequence-in the "one switch" condition, for example, repetition of LRRRRRR would produce reinforcement on $100 \%$ of the trials - they tended to vary their sequences. Barba and Hunziker (2002) showed, however, that variability was higher when it was directly reinforced than when reinforcement was based on proportions of switches, a finding consistent with that of Machado (1997).

The evidence from each of these methods supports the hypothesis that variability can be reinforced.
Measures. Variability must be interpreted with reference to a particular measure. The reason is that there can be high variability (and low predictability) at one level of analysis, but order (and certainty of prediction) at a different level. For example, the sequence 1, 1, 2, 2, 3, 3, 4, $4,5,5,1,1,2,2, \ldots$ has the same variance (a commonly employed measure of variability) as 2, 3, 2, 1, 5, 1, 4, 4, $1,1,3,2,5,2 \ldots$, but an analysis of conditional probabilities, in which the unit contains pairs of instances, indicates that the two sequences have different levels of variability. In general, high variability observed with one measure does not rule out the possibility of order observed with another, and vice versa. That is why evaluations of random-number generators often employ many different measures and, indeed, there are hundreds of different measures of the randomness of a sequence (Knuth, 1969). All behavioral studies make simplifying assumptions and rely on one measure, or at the most a few.

U-value has been the most commonly employed measure of operant variability (Evans \& Graham, 1980; Machado, 1989; Page \& Neuringer, 1985; Stokes, 1995). $U$-value measures the distribution of relative frequencies, or probabilities, of a set of responses. For a set of 16 possible responses, $i=1-16, U$-value is given by

$$
-\sum_{i=1}^{16}\left[\mathrm{RF}_{i} * \log _{2}\left(\mathrm{RF}_{i}\right)\right] / \log _{2}(16),
$$

where $\mathrm{RF}_{i}=$ the relative frequency (or probability) of each of the 16 responses. $U$-values approach 1.0 when relative frequencies approach equality, as would be expected over the long run from a random generator, and they approach 0.0 when a single instance is repeated.

Other measures include the percentage of trials, or frequency, at which variability contingencies are met (Page \& Neuringer, 1985); percentages of alternations versus stays (Machado, 1992); conditional probabilities of responses (Machado, 1992); the number or frequency of novel responses (Goetz \& Baer, 1973; Schwartz, 1982); the number or frequency of different responses (Machado, 1997; Schwartz, 1982); Markov analyses (Machado, 1992); and statistical tests of distributions of responses (Neuringer, 1986). All of the measures employed to date indicate that variability can be reinforced.

Responses and species. Generality has further been demonstrated by the use of many different responses and species. These include Siamese fighting fish (Betta splendens) swimming in quadrants of a tank (Roots, 2000), young chicks pecking keys (Neuringer, 2002), pigeons pecking keys (Page \& Neuringer, 1985), budgerigars singing songs (Manabe, Staddon, \& Cleaveland, 1997), rats pressing levers (Van Hest, van Haaren, \& van de Poll, 1989), porpoises swimming and jumping (Pryor et al., 1969), rhesus monkeys pressing levers (Sydney Reisbick, personal communication), children drawing and building with blocks (Holman, Goetz, \& Baer, 1977), and human adults and children pressing buttons (Saldana \& Neuringer, 1998; Stokes \& Balsam, 2001). There have been few attempts to compare species or responses, but 
in one study lowest baseline variability was observed when rats pulled trapezes, intermediate levels were observed when they pushed keys, and highest levels were observed when they pressed levers (Morgan \& Neuringer, 1990), with variability increasing in all cases when it was reinforced. In another study, operant variability was compared across rats and people, with similar effects observed in the two species (Neuringer, Deiss, \& Imig, 2000).

\section{Parameters}

We turn now to some parameters studied to date. Many other parameters, as yet unstudied, are no doubt also important-for example, motivation and types of reinforcers.

Age. Older people (see, e.g., Van der Linden, Beerten, $\&$ Pesenti, 1998) and older rats (see, e.g., Lamberty \& Gower, 1990) respond less variably than their younger counterparts when variable responding is reinforced or requested, but in most cases it is not clear to what extent the variability is elicited as opposed to reinforced. Neuringer and Huntley (1992) compared reinforced variability in 2-month-old (young) and 10-month-old (mature) LongEvans rats. Under Yoke contingencies, the young rats responded significantly more variably (two levers, four responses per trial) than the mature rats, which constitutes an elicited effect. When reinforcement was contingent on variability (lag 4), variability increased significantly in both groups, thereby overcoming the initial difference correlated with age. Thus, explicitly reinforcing variability in older animals caused them to respond no differently than younger animals did, a finding potentially relevant to real-world human aging. However, when Wells (1999) compared 8-month-old (mature) to 24-month-old (old) rats, although the mature rats responded more variably than the old rats did under Yoke contingencies, the difference was not statistically significant. Additional studies are needed, especially of whether reinforcing variable behaviors in older populations can facilitate learning and performance.

Gender. Male and female rats are equally sensitive to reinforcement of variability (Neuringer \& Huntley, 1992; van Hest et al., 1989). However, there was a tendency (not statistically significant) for males to respond overall more variably than females did, and because these studies did not control for differences in size and weight of subjects or for other aspects of motivation, additional studies are necessary.

Stage of training. Stokes and Balsam (2001) reported differences in variability as a function of whether variability was initially reinforced early or later in training (see also Gottlieb, 1992). Human subjects generated sequences of $10 \mathrm{~L}$ and $\mathrm{R}$ responses per trial on a computer keyboard under lag 0 and lag 25 contingencies. Under lag 0 , every trial ended with reinforcement; under lag 25, the current sequence had to differ from each of the previous 25 . When lag 25 was experienced at the very beginning of training, levels of variability at the end of training (when lag 0 was operative) were lower than they were when lag 25 was experienced after 50 trials of initial lag 0 . These results were interpreted to suggest that there is an "optimal period for setting variability levels" (Stokes \& Balsam, 2001, p. 181), but that general conclusion needs further testing, and a number of questions need to be answered. One has to do with the contribution of reinforcement frequency: Under lag 0 , every sequence leads to reinforcement, but reinforcement is intermittent under lag 25. Another has to do with whether learning to vary differs from learning to repeat with respect to optimal periods (see, e.g., Bouton, 1994).

Related to stage of training are effects of prior experience. Hunziker, Lee, Ferreira, da Silva, and Caramori (2002) showed that, for human subjects, if Var contingencies were experienced just prior to Yoke contingencies, levels of response variation during the Yoke phase were higher than they would have been if the Var contingencies had not been previously experienced. The time delay between Var and Yoke experiences mattered, with a long interval (3 months) producing only a small effect. Saldana and Neuringer (1998) also found effects of prior Var training on performances in Yoke (increased variability in Yoke), but the opposite did not hold; that is, training under Yoke had relatively little effect on Var. Hunziker, Caramori, da Silva, and Barba (1998) reported similar effects with rats. Thus, there may be an asymmetry: Reinforcement for varying has relatively strong influences, but reinforcement without regard to variations does not adversely affect learning to vary. These different effects are not surprising, given that Yoke contingencies are permissive-reinforcement is given whether or not responses vary-whereas contingencies under Var can be quite demanding. However, the results motivate a number of additional questions. One is whether effects of Var training differ from those observed when repetitions are reinforced. Another is whether direct reinforcement of variable behaviors can help animals or people to overcome the detrimental effects of prior noncontingentexperiences, such as those that produce learned helplessness (Seligman, 1975).

Environmental constraints. Stokes (1995) argued also that variability increases when behavior is constrained. The term "constraint" is ambiguous, because all reinforcement contingencies exert a form of constraint. In one study, Stokes (1995) compared different strategies of shaping leverpresses. Rats in one group were reinforced initially for pressing the lever with only the right paw (right only), and eventually for any press (with right or left paw, or with any other part of the body). The order of training was reversed for a second group; "any" first and then right only. Behavior sequences were more variable in the right-only-first group, leading Stokes (1995) to conclude that early constraints lead to high variability (see also Stokes, Mechner, \& Balsam, 1999). That is an important hypothesis, with many implications, but controls are needed before the results can be attributed to the contingencies. For example, "right only" and "any" differed in terms of reinforcement distributions as well as in the precision of the reinforcement operation; the "any" reinforcers were provided immediately and automatically by the programming equipment, whereas in 
the right-only condition, a human observer had to decide whether a response had occurred and then present the reinforcer, necessarily leading to longer and more varied latencies and a less consistent contingency. Furthermore, there are many cases in which increasing constraints, such as those due to a decrease in the number of responses per trial (Page \& Neuringer, 1985) or the requirement of exactly four responses on each of two keys (Schwartz, 1980), results in decreased variability. Thus, the contributions of response-definition, constraints, and environmental limitations must be explored further.

\section{Summary}

The generality of operant variability is shown by the robustness of the phenomenon across procedures, measures, responses, and species. As with other operant phenomena, parameters influence the effects.

\section{FUNCTIONS}

All operants, including variability, are functional: Operant responses produce reinforcing consequences. Because other behavioral competencies depend on it, operant variability is functional in a broader sense as well. Some of these functions will now be described.

\section{Operant Conditioning}

The potential of a reinforcer to change behavior is limited by the range of available variations (Hull, Langman, \& Glenn, 2001; Skinner, 1981) and, thus, whatever produces those variations contributes to the conditioning of new operants. Much work has been done to identify sources of variation (Balsam, Deich, Ohyama, \& Stokes, 1998; Segal, 1972; Staddon \& Simmelhag, 1971), but Neuringer (1993) was first to show that the same reinforcers that strengthen individual responses can also produce the necessary variability, and that these two functions of reinforcement may work simultaneously. Implied is that when an operant response is being conditioned, reinforcement works not only to strengthen an individual response, but at the same time to engender the variability from which the response emerges. (That is, variability is not solely a baseline phenomenon, elicited, or noise.) Concurrent reinforcement of variations and individual target sequences will be the topic of this section.

Pigeons in one experiment and rats in another were rewarded for varying among 16 possible response sequences (four responses per trial, L and R operanda, lag contingencies; Neuringer, 1993). Concurrently, one of the 16, designated as the target sequence, was reinforced whenever it occurred. Thus, reinforcements were given both for sequence variations and for a single selected target, a reinforced-variation-and-selection (RVS) procedure. When the target was "easy" (e.g., LLLL), it emerged rapidly from the baseline variations. Given that the target was always reinforced, reinforcers were obtained more frequently when the subjects did not vary and only repeated the target. When the target was "difficult" (e.g.,
LLLR), it was not learned. As in the choice experiment described above (Neuringer, 1992), the relative values, or difficulties, of target versus variations governed the probability that the target would be learned.

However, even difficult targets could be conditioned under the RVS procedure, as was shown by another experiment in the same series (Neuringer, 1993). Sequence variations were again reinforced concurrently with a difficult target, RRLR, but now the frequency of reinforcements for varying was systematically decreased: Satisfying the variability contingency was intermittently reinforced according to a VI schedule, and over sessions reinforcement for varying became less and less frequent. This technique resulted in acquisition of even the most difficult target sequence, whereas a control group, reinforced for the target alone (without the concurrent reinforcement for variations), never learned.

Reinforcers were obtained more frequently in the RVS condition than in the control condition (since the RVS subjects were rewarded both for varying and for emitting the target sequence), and the additional reinforcers might have generated greater motivation to respond (Killeen, 1981). Thus, motivation, not the direct reinforcement of variability, might have been responsible for the RVS effect. To test this possibility, Neuringer, Deiss, and Olson (2000) reinforced three groups of rats for emitting a particularly difficult target sequence: LLRLR. The experimental group was reinforced concurrently for sequence variability on the average of once per minute (VI $1 \mathrm{~min}$ ). The target-only control group received no reinforcements other than for the LLRLR target. A third group was yoked to the experimental animals-reinforced once per minute for any sequence, variability not requiredand, as for the other two groups, whenever the target occurred. If the additional reinforcement was responsible for the facilitated learning under RVS, then, because the experimental and yoked groups received equal reinforcements, they should have learned equally well, but that was not the result. Only the experimental group learned to emit the LLRLR target. The yoked controls responded at high rates throughout the experiment, but they did not vary sufficiently to make contact with the target contingency, and never learned. The target-only control group received insufficient reinforcement, and their responses extinguished. Therefore, the added reinforcers helped to maintain motivation, as suggested above, but reinforcement of variations was necessary for learning of a difficult sequence. The RVS procedure maintains both high motivation and high variation, and the two together result in the acquisition of difficult-to-learn responses.

Grunow and Neuringer (2002, Experiment 2) studied one possibly important parameter of the RVS procedurenamely, the level of variability. When a difficult target response is being trained, does it matter whether reinforced variability is low, intermediate, or high? As described above, each of four groups of rats was reinforced for variable response sequences: the first group for high variability, the fourth group for low variability, and the other 
two for intermediate levels. In other ways, the procedure was similar to that of the Neuringer, Deiss, and Olson (2000) RVS contingencies just described, with a single, difficult target sequence always reinforced. The results were that the higher the level of reinforced baseline variability, the more quickly the target was learned. Thus, variability levels indeed mattered, and if the just-reported effect is found to be general, it will be most helpful to reinforce high variability when difficult-to-learn new responses are being shaped. Other parameters requiring study are class definitions-which responses are included in the class of acceptable variations - and number of instances within the class.

One additional effect should be noted. When variations among a set of responses are reinforced, if one variant is selected for extinction (no longer reinforced), the frequency of that instance decreases. Neuringer (1993) showed this for pigeons responding under a lag-variability schedule. When one of the 16 sequences was no longer reinforced (with no other change to indicate its status), the frequency of that sequence decreased. Thus, reinforcement of variations provides the baseline to select for (i.e., strengthen) and select against (i.e., weaken) individual instances.

These findings have potentially important implications. First, as has been indicated, the same reinforcers that strengthen particular instances can also reinforce variations. That a binary event-reinforce or not reinforcesimultaneously affects variations and selected instances indicates both the power of reinforcement and a conceptual problem. How reinforcers exert such diverse effects is a question to which we will return.

Second, shaping procedures may implicitly reinforce baseline variations. An approximation to a goal response is reinforced for a period of time, but eventually reinforcement is withheld until a response closer to the goal is obtained. It is commonly thought that withholding reinforcement, or mini-extinction, induces the necessary variations for shaping to proceed, as has been indicated above. An alternative, suggested by the present studies, is that organisms learn both to vary (within a set) and to repeat (selected instances) until one instance is always reinforced. That is, the process of shaping might involve reinforcement both of variations (generally done implicitly) and of targeted instances. If so, a useful contributor to the shaping process might be explicit reinforcement of variations.

Third, reinforcement of variations could facilitate acquisition of other competencies, such as cognitive and motor skills. Siegler (1996) has shown, in an important series of studies, that children's acquisition of complex cognitive skills is correlated with high variability. Another example is that when motor skills are practiced in a variable manner, high quality of performance results (Manoel \& Connolly, 1995, 1997; Mechner, 1992; Schmidt $\&$ Lee, 1999). However, it is rare that variability in these domains is directly reinforced as part of the training or educational process.
Fourth, the RVS procedure might be used in therapeutic settings to modify maladaptive and self-injurious habits. Therapists sometimes employ noncontingent reinforcement procedures to weaken undesirable behaviors (Vollmer, Iwata, Zarcone, Smith, \& Mazaleski, 1993). The reasoning is that many maladaptive behaviors are maintained by unintended consequences and that noncontingent rewards may compete with those consequences, thereby lowering the strength of the unwanted habit. For example, when a staff person's attention to self-injurious behavior helps to maintain it, providing attention noncontingently may decrease the strength of the unwanted target behavior. However, the RVS procedure might accomplish the same goal more effectively and without undesirable side effects (Vollmer, Ringdahl, Roane, \& Marcus, 1997). Noncontingent rewards sometimes result in unwanted "superstitious" behaviors, and that would be avoided under RVS. Furthermore, RVS would help to provide the set of baseline behaviors necessary to establish desirable new responses.

Finally, RVS procedures may help to gain control over, and eventually to weaken, other undesirable target behaviors. The target can be incorporated within a set of more desirable responses - a new operant class established with the target as one member-and variable instances reinforced. Once variable responding is successfully maintained, including the undesirable target response, the latter can be weakened by selectively withholding reinforcement.

Summary. The conditioning of operant responses depends on both baseline variations and selection of successive approximations to a goal response. The same reinforcers used to select the approximations can reinforce baseline variations, thereby defining the precise set of appropriate variants and the desired levels of variation. Such concurrent reinforcement of variations and selections may facilitate the acquisition of difficult-to-learn operants, and selective withholding of reinforcement may weaken undesirable instances.

\section{Creativity and Problem Solving}

Behaving in a novel, unusual, or nonrepeating manner is part of creative activities (Campbell, 1960). Novelty and variation may arise through combinations of previously learned behaviors (Epstein, 1996), transformations of these behaviors (Boden, 1994), direct reinforcement of variability, as described above, or some combination thereof. Of course, neither novelty nor variability alone suffices for creative output - the throw of the dice or the toss of a coin are not creative, nor are haphazard strokes of a paintbrush on a canvas-but without variation, creativity is low. Whether the variability required for creativity can be reinforced is relevant to whether creativity itself can be reinforced. The latter question has led to contentious debate, with some arguing that reinforcement facilitates creativity (Cameron \& Pierce, 1994; Eisenberger \& Cameron, 1996; Stokes, 2001; Winston \& Baker, 1985) and others focusing on detrimental effects of reinforcement (Amabile, 1983; Deci, Koestner, \& Ryan, 1999). 
The debate goes well beyond the present discussion, but some of the research described above is relevant. First, reinforcement contingent on variability generates higher levels of behavioral variability than does reinforcement contingent simply on responding, as has been shown by the many studies described above in which Var contingencies were compared to Yoke contingencies. Similarly, precise reinforcement contingencies matter for creativity (Cameron \& Pierce, 1994; Lepper \& Henderlong, 2000). If any product, rather than only creative ones, is reinforced, creativity tends to fall (just as variability decreases under yoke conditions). Second, approach to or anticipation of reinforcement interferes with operant variability (Cherot et al., 1996; McElroy \& Neuringer, 1990; Neuringer, 1991), which again might be related to effects on creativity when an individual focuses on the reinforcer. Note, however, that although variability decreases with proximity to the reinforcer, overall levels of variability are much higher when variability is reinforced than when it is not. The same may be true for creativity as well; anticipation of reward may interfere with creative output to some extent but, at the same time, high creativity may depend on its reinforcement.

Operant variability may be important for problem solving as well. Here, too, there is disagreement as to whether training is helpful or not. Early studies by Maier (1933) indicated that it is, and these found support in later work (e.g., Lung \& Dominowski, 1985). Some studies showed that those individuals most likely to "break the set" are also most likely to solve a problem (Dover \& Shore, 1991). However, a number of studies found that practice had no influence (Duncan, 1961).

Arnesen (2000) explored a related phenomenon with a rat model. One group, Var, was reinforced for variable interactions with novel objects, and another group, Yoke, was reinforced independently of such interactions. Each Var rat received training with 12 different objects, one per session. For example, in the presence of a soup can, the rat was reinforced the first time it entered the can, but thereafter a different response was required, such as rolling it, climbing on it, and so forth. This procedure is similar to that of Goetz and Baer's (1973), described above, except that objects changed from session to session. Rats in the Yoke control group had identical experiences in an adjoining identical chamber that contained identical objects, but now rewards were not contingent on object manipulation. After this training, the rats were tested in a different room, with 30 never-before-experienced objects scattered on the floor. Hidden in each was a small piece of food. When compared to their Yoke controls, the Var animals were more active during this test, explored more, manipulated objects more, and, most importantly, discovered significantly more food pellets. Another control group received no preliminary training with objects, and their performance in the test session did not differ from that of the yoked controls. Therefore, when rats were reinforced for variable interactions with objects, the result was an increased tendency to explore, manipulate, and discover new sources of reinforcement. It would be of considerable interest to determine whether a similar effect occurs with children.

\section{Assessment and Treatment of Psychopathologies}

Abnormal levels of behavioral variability characterize some human psychopathologies, as has been shown by clinical observations, as well as by research with human subjects and animal models. Few studies have attempted to reinforce variability directly in such problem populations. The main question is whether reinforcement can modify abnormal levels of variability in the direction of normalcy, and whether such changes might have associated beneficial effects.

Autism. Stereotyped responding characterizes individuals with autism (Hertzig \& Shapiro, 1990). For example, Baron-Cohen (1992) asked children with autism and others in a control group to hide a penny in one hand so that the experimenter could not guess which hand held the penny. Those with autism were more likely than those without to generate a simple, predictable pattern, such as repeatedly switching back and forth from left to right hands. Such repetitive responding can be maladaptive. For example, Mullins and Rincover (1985) asked children with and without autism to pick one of five cards. Food was occasionally found in a cup behind the card. Each of the five cards was a discriminative stimulus for a different schedule of reinforcement, including continuous reinforcement (CRF, every choice reinforced), FR 2 (fixed ratio 2 , in which every second choice was reinforced), FR 4 (every fourth choice reinforced), FR 7, and FR 11. Control participants sampled all five alternatives and quickly learned to choose the most frequently reinforced CRF card. Autistic children sampled only a limited number of cards and therefore often preferred a nonoptimal alternative. Thus, children with autism tend to respond less variably than normal children, and this can result in failure to adapt.

Miller and Neuringer (2000) asked whether direct reinforcement could influence the variability of individuals with autism. Sequences of responses on two large buttons were first reinforced probabilistically and independently of variability (Prob phase), after which reinforcement was contingent upon satisfying a threshold variability contingency (Var phase). The autistic participants' variability was significantly lower than that of matched controls during both Prob and Var phases, but when variability was reinforced, its levels increased significantly in both groups. This is an important finding because it suggests that direct reinforcement of variability may help to modify some of the behavioral stereotypes that are characteristic of autism. Lee, McComas, and Jawor (in press) offer support for this hypothesis. Three individuals with autism were reinforced for varied verbal responses to social questions; the responses had to be appropriate given the context. Variability increased in two of the three individuals. In a related procedure, Baker and Koegel (1999) reinforced children with autism for varied social interaction in a game-playing context. Although the reinforcement was permission to engage in 
the stereotyped behaviors preferred by the child, the result was increased social interactions, both during the period of the experimental manipulation and in 1- and 2-month follow-ups. These three studies, taken together, suggest that reinforcement of varied behaviors may facilitate modification of nonfunctional ritualistic and stereotyped behaviors.

Depression. Depression provides another example of maladaptive consequences of low variability. Lapp, Marinier, and Pihl (1982) observed that depressed women produced fewer alternative solutions to hypothetical problems than did nondepressed women. Channon and Baker (1996) found that attempts by moderately depressed college students to identify faults in a series of interconnected circuits were less varied and less successful that were those of nondepressed controls. Horne, Evans, and Orne (1982) found that, when depressed patients were asked to generate a random sequence of numbers, their responses were less variable than were those of controls. These experimental results are consistent with clinical observations.

Can depressed individuals be rewarded for increasing variation? Hopkinson and Neuringer (in press) divided college students into mildly depressed and nondepressed groups, on the basis of the CES-D scale, a paper-andpencil self-evaluative index of depression. In the first of two phases, sequences of responses on a computer keyboard were reinforced independently of variability (Prob), following which only highly variable sequences were reinforced (Var). Under Prob, depressed students responded significantly less variably than did the controls, in agreement with previous findings. Under Var, variability increased significantly in both groups. Indeed, by the end of the experiment, depressed and nondepressed subjects were responding equally variably. (Instructions were also effective in increasing variability in the depressed participants.) Thus, even in a simple computer-game environment, mildly depressed subjects manifested lower variability than controls did, but direct reinforcement modified their behavior to control levels. Can reinforcement of variability be used in real-world conditions to help depressed individuals? An affirmative answer comes from cognitive behavior modification procedures that work to increase alternative solutions to problems (Beck, 1976). A more general conclusion from these studies of autistic and mildly depressed individuals is that directreinforcement-of-variability procedures may benefit those who manifest abnormally low levels of variability.

Attention Deficit Hyperactivity Disorder (ADHD). ADHD may provide an example of the opposite state: abnormally high levels of variability (Barkley, 1990). Wultz, Sagvolden, Moser, and Moser (1990) proposed that the spontaneously hypertensive rat (SHR) may serve as a good animal model of ADHD; the SHR shows many of the behavioral characteristics of that disorder, including hyperactivity, poor learning ability, poor ability to tolerate delayed reinforcement, and poor self-control.
The next study to be described asked about operant variability in SHRs.

Mook, Jeffrey, and Neuringer (1993) compared variability of SHRs and of a control strain, Wystar Kyoto (WKY), under two conditions, Prob and Var. The main result was that SHRs tended to respond variably whether or not the contingencies required it, consistently with the results from the human ADHD literature. When variability was reinforced, the two strains responded at approximately equally high levels of variation. These results were confirmed in two other studies (Hunziker, Saldana, \& Neuringer, 1996; Mook \& Neuringer, 1994). Thus, control rats varied only when the contingencies required variations, whereas SHRs varied whether or not the contingencies required it. As might be expected from these results, when reinforcement depended on repetitions, the SHRs performed poorly (Mook et al., 1993).

Another similarity between SHRs and ADHD humans was seen when amphetamine, a drug that has effects similar to those of Ritalin, often prescribed for ADHD, was administered (Mook \& Neuringer, 1994). When repetitions were required for reinforcement, amphetamine improved performances by the SHRs so that they were as accurate as saline-injected WKYs, a finding again consistent with those in the ADHD literature. These studies support SHRs as a model of ADHD and suggest that additional attempts should be made to assess whether or not individuals with ADHD are less able to change levels of variability than are controls. Although, as indicated above, there is some evidence in support of this hypothesis (Barkley, 1990), when Saldana and Neuringer (1998) directly compared human ADHD and control participants' operant variability within the context of a computer game, no differences were observed. Most of those with ADHD were maintained on Ritalin and other drugs, however, a fact unfortunately not documented in the original article.

\section{Drug Effects}

Although many drugs are known to influence behavioral variability (Brugger, 1997), rarely have attempts been made to analyze, explain, or identify the sources of the effects. As will be discussed below, operant variability can result from the memory-based process of remembering previous responses and then behaving differently, or can result from responding stochastically, a process that appears to be quite different. McElroy and Neuringer (1990) attempted to distinguish the effects of alcohol on memory from its effects on stochastic processes in a study in which one group of rats was reinforced for varying four-response sequences under a lag 5 contingency (Var), and another was reinforced for repeating a single sequence, LLRR (Rep). Ethanol injections of 0.75 and $2.0 \mathrm{~g} / \mathrm{kg}$ affected Var and Rep performances differently. Whereas the Rep animals were much less able to satisfy the LLRR contingency at both doses, the Var rats responded variably whether or not alcohol had been administered. Similar results were obtained by Cohen et al. 
(1990) with a multiple schedule in which each rat served as its own control. In the same animals and within the same sessions, alcohol degraded Rep performances but left Var intact. Thus, alcohol appears to interfere with a task that requires working memory, as presumably is the case for the Rep task, but not with one that reinforces variability. The memory-interfering effect of alcohol might account for why alcohol also degrades radial-arm maze performance (Devenport, 1983).

Alcohol also affects choices to vary or to repeat. For example, Gorka (1993) reinforced rats concurrently for repeating and varying, and monitored percentage of choices as a function of time following injection of ethanol. In the control sessions, when the rats were injected with saline, the probabilities of varying and repeating remained approximately constant across the 45-min session, with a slight bias in favor of varying. After injection with 1.5 $\mathrm{g} / \mathrm{kg}$ of ethanol, however, there was a significant increase in choices to vary at the beginning of the session, and then a later decrease to below $50 \%$. That is, alcohol led initially to a markedly increased preference for varying, but later to a preference for repeating (Figure 5). This research should be replicated and extended, especially because the contingencies "pressured" the animals to remain at approximately $50 \%$ preference, but the suggestion is that alcohol's effects on response variability may depend in part on the duration of its influence. A more general implication is that drugs may affect repeated and variable operants differently, and that effects depend on time following administration.

\section{Summary}

Reinforced variability may contribute to many types of learning, including operant shaping and acquisition of cognitive and complex motor skills. Similarly, creativity and problem solving depend on variable responding, and in these areas as well, explicit reinforcement of variations may be functional. Abnormal levels of variability are sometimes caused by psychopathologies and drugs, and operant variability may be useful in these areas in two ways: first, as a way of assessing the variability that is possible, and second, to modify variability toward more normal levels. Studies show that individuals with autism and mild depression vary less than do controls, but that explicit reinforcement of variability moves the levels towards normalcy. Other studies show that drugs such as alcohol may affect repeated and variable operants differently.

\section{EXPLANATIONS}

Two questions must be answered in order to explain operant variability. First, what are the sources of variability that permit its reinforcement? Second, how is it maintained? Related questions, including how instances become members of a set from which variations emerge, and whether operant variability is a generalizable competency, will also be considered.

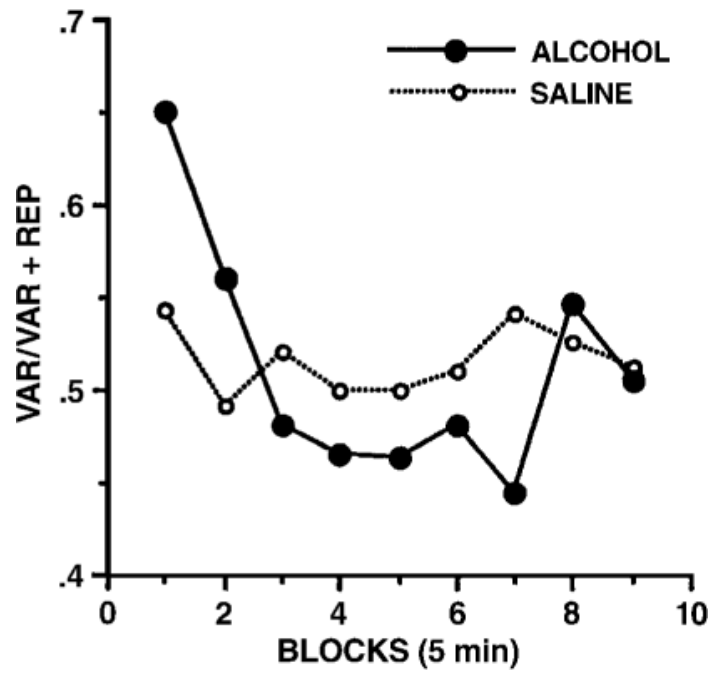

Figure 5. The relative frequency of variations by rats as a function of 5-min blocks of time since the beginning of the session. The open circles represent performance after the rats were injected with saline, indicating a slight tendency to vary more than repeat, but one that is approximately constant throughout the session. The filled circles represent performance after injection with $1.5 \mathrm{~g} / \mathrm{kg}$ ethanol, indicating a much higher likelihood of varying soon after the alcohol is injected, followed by a decrease in variations to below $50 \%$, and then a return to baseline at the end of the session. Group averages are shown. Data are from Gorka (1993).

\section{Origination From Nonoperant Sources}

Skinner (1966) reasoned that operant responses originate from nonoperant sources, with reinforcement contacting a behavior, shaping and strengthening it, only after the behavior had occurred for reasons other than reinforcement. To put it simply, a behavior must occur before it can be reinforced (Balsam \& Silver, 1994). I will consider three initial sources of variable behavior: endogenous variability, noncontingentenvironmental events, and extinction. These appear to parallel evolutionary processes and they, too, will be identified.

Endogenous variability. All physical phenomena vary, and behavior is no exception. This fact can provide the opportunity for reinforcement to select particular levels of variation. Examples of spontaneous variability include spontaneous alternations in mazes (Dember \& Richman, 1989), nondifferentially reinforced variations in operant responses (Guthrie \& Horton, 1946), and exploration (Archer \& Birke, 1983). Endogenous mutations provide a parallel in genetics. Base-pair changes in DNA molecules occur spontaneously, intron-bearing genes mix and match segments to encode new proteins, jumping genes introduce new DNA sequences, and, at the level of the chromosome, random crossings and assortment occur as a natural part of reproductive meiosis. These sources of random genetic change occur independently of external influences. 
Noncontingent events. Noncontingent events, sometimes referred to as chance, accidents, or luck, also affect behavior. One happens upon a particular passage in a book that leads to a new thought; one happens to sit next to an interesting woman or man on an airplane, which leads to marriage (Bandura, 1982). In science as well as in everyday life, accidents, or serendipity, are an important part of the process of discovery (Beveridge, 1957). In each of the cases presented above, an accidental event leads to new behaviors that are then strengthened by consequences. The same is potentially true for variability itself. Particular levels of variability might occur for adventitious reasons, but then become functionally related to consequences.

That organisms are particularly sensitive to such unanticipated, noncontingent events is shown by two basic principles of learning: habituation and Pavlovian conditioning. Organisms attend preferentially to unexpected events and stop attending, or habituate, to repeated ones. The conditioning of new Pavlovian responses depends, according to influential theories, upon unexpected reinforcing events (e.g., Rescorla \& Wagner, 1972).

Noncontingent events, such as X-rays and other processes that can act as mutagens, play an important role in genetic variation as well. The particular genes affected and the type of changes are random; there is no necessary relationship, or contingency, between the external event and the affected gene. However, sensitivity to mutations is not random, but has been selected, presumably because it is functional. Thus, in both behavior and genetics, sensitivity to noncontingent events is conserved.

Extinction. Extinction increases behavioral variability, at least to some extent, as was described above. Distance in space or time from the reinforcer does the same (Cherot et al., 1996). A possible parallel at the genetic level involves high levels of deprivation, at least in simple life forms. For example, when E. coli bacteria are deprived of nutrients, unusually high mutation rates are observed. The exact mechanism by which such hypermutations are induced is not known, but they increase the likelihood that a population can survive such environmental insults (Moxon, 1997). That is, deprivation-induced variability is functional at the genetic level. (The behavioral evidence regarding long-term deprivation is less clear, some studies showing increased variability and others increased stereotypy; e.g., Carlton, 1962; Senkowski, Vogel, \& Pozulp, 1978).

Endogenous variations, external noncontingentevents, and withholding of reinforcement are three sources for the variability that precedes control by reinforcement. But once control by reinforcement is established, a different question emerges: What behavioral processes are involved when variability is maintained as an operant? The sources just discussed generate uncontrolled, nonspecific, and often short-lived variability and cannot explain the precisely controlled, operant type.

\section{Operant Variability Processes}

Three processes are hypothesized to underlie operant variability. The first involves the use of random environ- mental events, such as the toss of a coin. The second involves memory for responses, such as when an animal learns not to return to previously visited locations. The third will be referred to as an endogenous stochastic generator.

Random environmental events. Throughout history, many cultures have used random events to influence choices and decisions, such as throws of dice and random selections of sticks, cards, bones, or organs. Today, coin flips are used by a referee at the beginning of a game to decide which team kicks the ball; a computer's randomnumber generator is employed by scientists to avoid biases in assigning subjects to experimental groups; and aleatoric events are sometimes employed in modern art, music, and literature. The Dice Man by Rhinehart (1998) provides a fictional example in which the protagonist, bored with life, writes a number of possible actions on slips of paper, and then periodically selects one blindly and behaves accordingly. These examples show that random external events may be used to avoid biases, engender unlikely responses, and break out of behavioral niches.

Memory-based variability. Many methods used to study operant variability differentially reinforce nonrepetitions, at least over the recent past. Therefore, one strategy that subjects can employ is to cycle through few responses or sequences, especially when the variability contingencies are relatively permissive. For example, under a lag 2 contingency, cycling repeatedly through three sequences will result in $100 \%$ reinforcement. Animals and people sometimes do this, apparently basing their current response on memory for, or discriminative control by, the just emitted responses. One such instance was shown by Schoenfeld et al. (1966), who rewarded rats for IRTs that fell into one of 10 interval classes only if the current IRT differed from the interval class of the previous response. The rats came to alternate between long and short IRTs, thus behaving systematically to meet this lenient variability contingency. Machado (1993) studied an analogous effect, having pigeons peck $L$ and $R$ keys under a frequency-dependent variability contingency related to Shimp's (1967) procedure, described above. When the trial unit consisted of two responses, the birds developed memory-based repetitive sequences, such as RRLLRRLL, and thereby satisfied the variability contingency. When the trial unit was three responses in length, the birds did not develop the optimal fixed pattern of RRRLRLLL - which was too complex - but, instead, developed "random-like behavior" (Machado, 1993, p. 103). Thus, a memory-based strategy was employed when it was possible; but when the memory load became too high, stochastic responding emerged. A similar pattern was seen when variability of budgerigar songs was reinforced (Manabe et al., 1997). Under lag 1 contingencies, the birds sang mainly two songs; under lag 2, they increased to three songs. Only when the lag was increased to 3 did song diversity increase appreciably in one of the birds. In radial-arm mazes as well, when contextual cues are available, memory again appears to play an important role, leading to much lower probabil- 
ities of repetition than would be expected from a stochastic generator (McElroy \& Neuringer, 1990).

Note that under lag schedules, memory-based strategies result in higher reinforcement rates than responding stochastically does. The reason is that a stochastic generator repeats by chance. That animals and people often do not employ memory-based strategies probably indicates the difficulty of remembering long sequences, and possibly other advantages of unpredictable responding.

If a memory strategy is the only way to satisfy variability contingencies, then it can be used even when the memory requirements are demanding, as was shown by Neuringer and Voss (1993). Human subjects were trained to generate on a computer keyboard a chaotic-like sequence of responses that matched iterations of the logisticdifference equation:

$$
\mathrm{R}_{n}=t * R_{n-1} *\left(1-R_{n-1}\right),
$$

where $\mathrm{R}_{n}$ refers to the $n$th iteration in a series, with $\mathrm{R}$ between 0.0 and 1.0, and $t$ is a constant between 1.0 and 4.0. When the $t$ parameter approaches 4.0, iterations of this equation result in very "noisy" sequences. As with other chaotic processes, however, there is an orderliness underlying the surface noise. In this case, if responses are autocorrelated, with $\mathrm{R}_{n}$ drawn as a function of $\mathrm{R}_{n-1}$, the resulting function is a parabola. In the Neuringer and Voss (1993) study, following each response, college students were shown how close their predicted values were to that from iterations of the logistic-difference model. In one experiment, the students made digital predictions; in another, they placed a pointer along a horizontal line, and this analogue response was then translated into digital values for computation. With training, the students became increasingly adept at responding in chaotic-like fashion - the students' predictions matched closely the iterations of the logistic function - and their autocorrelations became parabolic. The left column in Figure 6 shows performances by 4 subjects (rows) early in training that is not well approximated by the logistic-difference parabola, and the right column shows performances at the end of training, in which $r^{2}$ values were $.72, .99, .98$, and approaching 1.0. How was this accomplished? Since each iteration in the logistic-difference sequence is based on the prior output - that is, the sequence is generated by a deterministic, although nonlinear, process-it was hypothesized that the human subjects' chaotic-like responses were also based on memory for prior responses. To put it simply, the subjects may have memorized a long series of pairs in the form "If the previous response was value A, then the current response must be value B," as Metzger (1994), Ward and West (1994), and Ward (2002) have suggested.

To test whether subjects in fact relied on memory for prior responses, Neuringer and Voss (2002) systematically slowed responding by interposing pauses-that is, by requiring an IRT. As IRT increased, the difference between the subjects' sequences and the model's chaotic
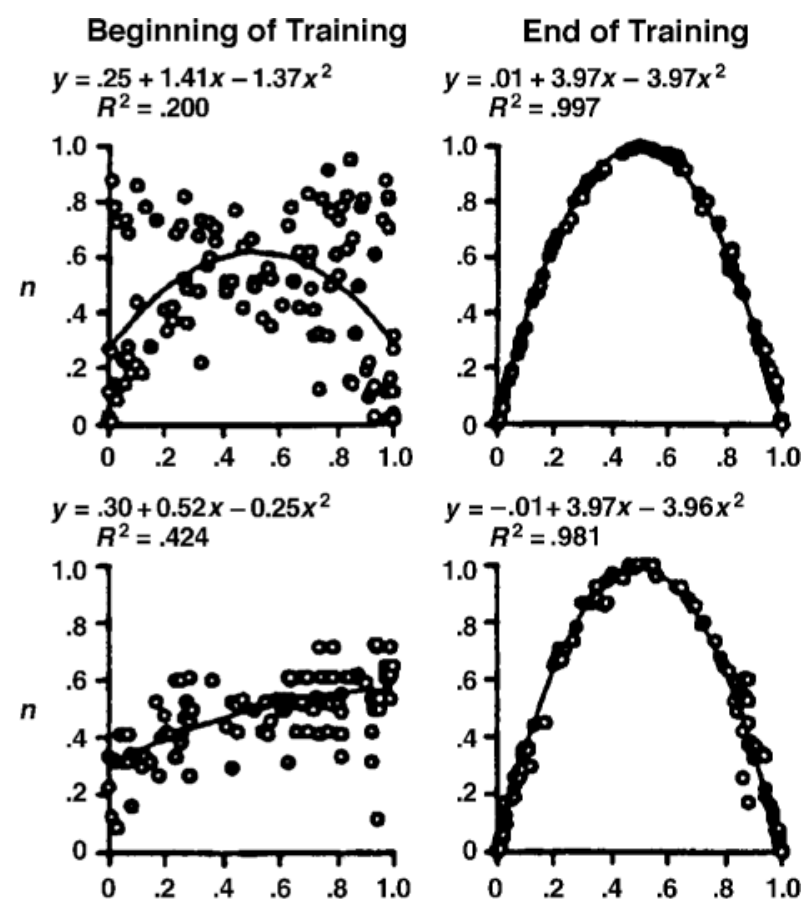

$y=-.01+3.97 x-3.96 x^{2}$
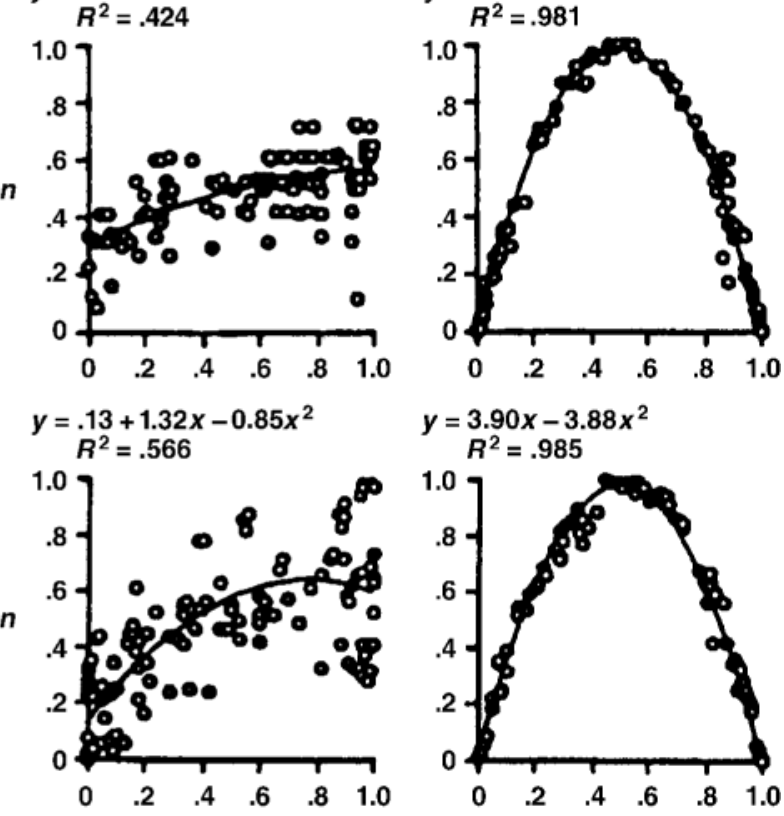

$y=3.90 x-3.88 x^{2}$

$R^{2}=.985$
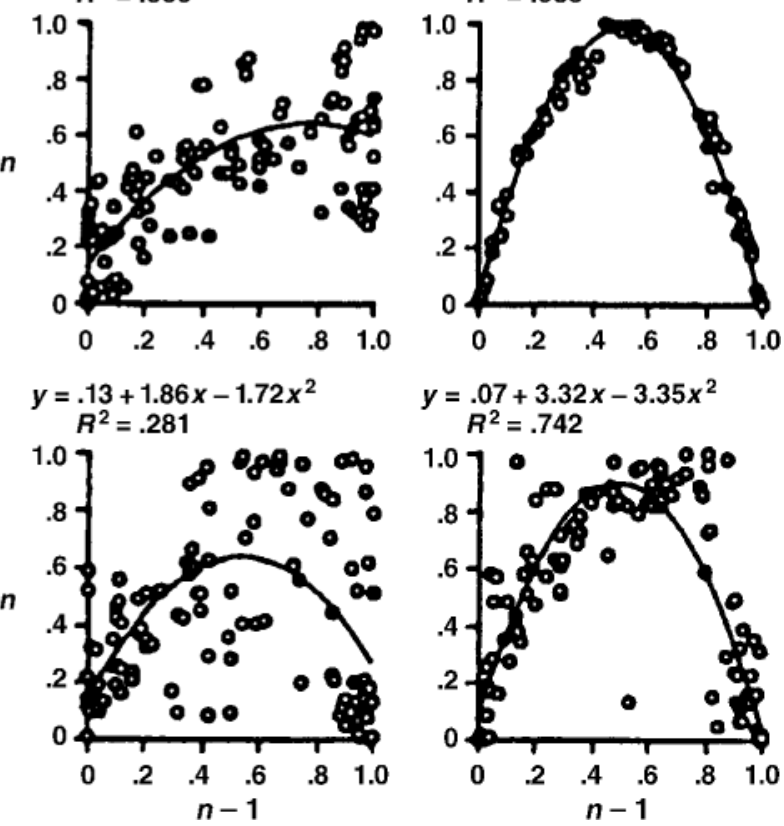

$y=.07+3.32 x-3.35 x^{2}$ $R^{2}=.742$

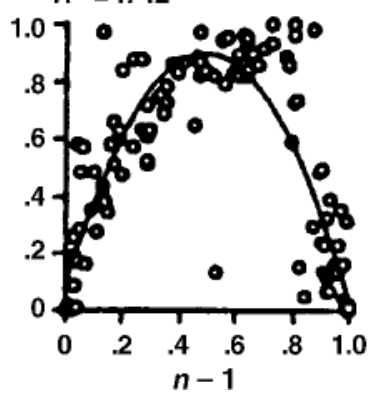

Figure 6. Autocorrelations (responses on trial $n$ on the $y$-axis as a function of $n-1$ on the $x$-axis) for 4 human subjects at the beginning (left column) and end (right column) of training in Neuringer and Voss (1993). Equations for the best-fitting curve and amount of variance accounted for $\left(r^{2}\right)$ are shown at the top of each panel. To the extent that the circles (responses) approximate the equation $y=4.0 x-4.0 x^{2}$, the subjects were responding in a way that matched the logistic-difference chaotic function. From “Approximating Chaotic Behavior," by A. Neuringer and C. Voss, 1993, Psychological Science, 4, p. 115. Copyright 1993 by the American Psychological Society. Reprinted with permission. 


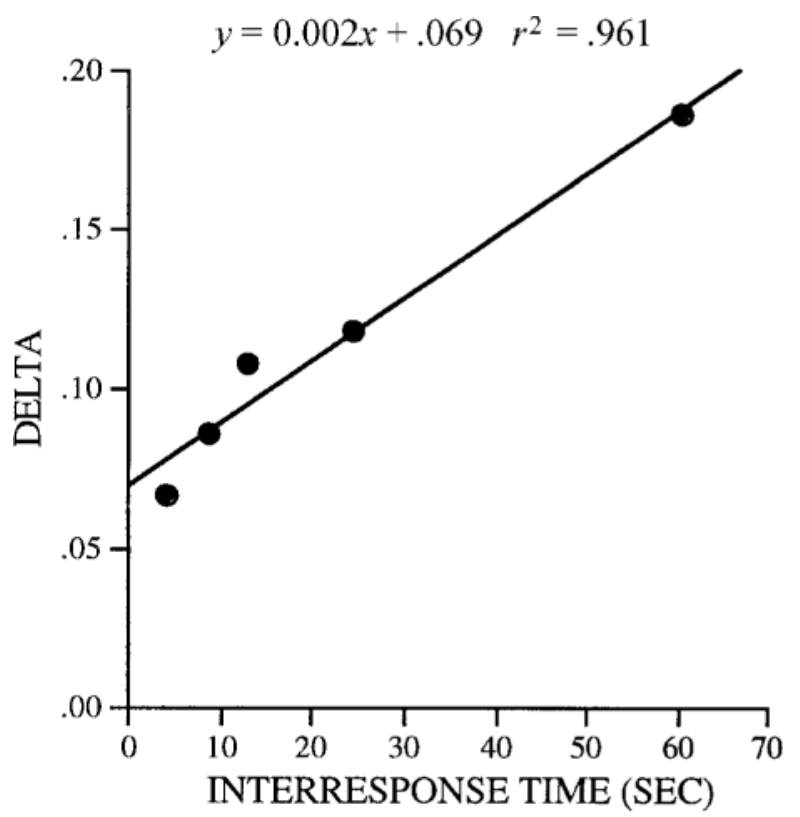

Figure 7. Approximation to a chaotic sequence (given by delta) as a function of the imposed interresponse times. Shown are the averages of 5 subjects.

output increased, and the underlying parabolic structure was broken. That is, when pauses increased, subjects were less and less able to approximate the model's chaotic distribution (see Figure 7). The important point here is that highly variable responses, which in this case are chaotic in nature, can be based on memorial processes.

Endogenous stochastic generator. The evidence to be reviewed next indicates that animals and people can respond stochastically, and that this competency is based neither on chance environmental events nor on memory for prior events or responses. It is impossible to prove that an endogenous stochastic process underlies operant variability; that would be analogous to proving the null hypothesis. However, much evidence is consistent with the stochastic hypothesis. Included are the facts that memory limitations do not permit adequate responding under many variability contingencies; that response distributions match those expected from a stochastic generator; that changes in parameter values affect operant variability as they would a stochastic generator; and that interference with memory processes leaves stochastically based operant variability unaffected. As a whole, the evidence is consistent with an endogenous stochastic source of operant variability.

Memory limitations. One type of evidence is negative in character, and is based on the presumed inability of memory for prior responses or sequences to satisfy some types of variability contingencies. Two examples were given above (Machado, 1993; Manabe et al., 1997), and another was provided by a lag 50 contingency in Page and Neuringer (1985). In the latter case, the sequence in the current trial (eight responses across L and R keys) was required to differ from those in each of the previous 50 trials, including, at the beginning of a session, the last 50 trials from the previous session. Pigeons responded well under these contingencies and in a way similar to that of a stochastic model. It is unlikely that pigeons can remember each of their previous 50 eight-response sequences.

Response slowing. As was shown above for chaoticlike responding, performances are often degraded by delays imposed between a stimulus and a response, presumably because of limitations in working, or short-term, memory (Berryman, Cumming, \& Nevin, 1963; Williams, 1971). Neuringer (1991) relied on this effect to test whether memorial processes could explain the generation of variable sequences under lag schedules. Two groups of rats were reinforced for $\mathrm{L}$ and $\mathrm{R}$ leverpresses, producing four responses per trial. One group (Var) was trained under a lag 5 contingency, and the other (Rep) was trained to a single sequence of responses, LLRR. It was assumed that accurate Rep performance depended on working memory. After performances had stabilized, IRTs were systematically varied by the imposition of timeouts of 0.5 to $20 \mathrm{sec}$ between consecutive responses in the sequence. During these forced pauses, the chamber was dark, and responses were not counted toward completion of the sequence. If the animals had used the previous response(s) as a discriminative cue for the current response, then performances should have been degraded by these interpolated pauses.

The results were that, for the Rep group, as the required pauses increased from 0 to $6 \mathrm{sec}$, percentages of correct sequences were relatively unaffected, but at pauses greater than $6 \mathrm{sec}$, probability of a correct LLRR sequence fell sharply. This pattern of results suggests involvement of working memory of prior responses, with the rats able to remember responses within a 6-sec window. The Var group's results were quite different: As pauses increased between 0 and $6 \mathrm{sec}$, the rats were more and more likely to satisfy the variability contingencies, and with pauses greater than $6 \mathrm{sec}$, percent correct remained at a high and constant level. Thus, the Var group's results were opposite to those of the Rep group and opposite to those predicted from a memory-based strategy. Similar results, showing that operant variability increases or is maintained as responding is slowed, have been reported by Morris (1987) with pigeons and by Baddeley (1966) with random response generation by human subjects. The results reported above concerning alcohol's effects on variability and repetition were also consistent with those of Neuringer (1991).

Memory for prior responses may not have controlled the Var group's performance, but why did variability increase? Each of three answers is consistent with a stochasticgenerator interpretation. Weiss $(1964,1965)$ hypothesized that random responding requires that current responses be independent of previous ones; memory for, or control by, prior responses interferes with such indepen- 
dence, and therefore, pauses would be expected to facilitate variability. A second possibility is that at short IRTs, animals tend to repeat responses on the same operandum. Blough (1966) found this in pigeons and excluded such double pecks from his analyses because they appeared not to be under the control of reinforcement contingencies. Morris (1987) also found a tendency for birds to repeat when no interresponse timeouts were imposed. A third hypothesis is that there were two contributors to the observed variability. One was a stochastic process controlled by reinforcement, and the other elicited variability generated by interposition of pauses. The high variability elicited as an effect of slowed responding is a general phenomenon, supported in many other cases. According to this interpretation, operant variability in the Var group was governed by a stochastic-based process, operant repetition in the Rep group was governed by a memorybased process, and pauses elicited variability under both contingencies. Thus, Rep performance was interfered with, whereas Var was facilitated. Each of these hypotheses is consistent with the conclusion that memory for (or discriminative control by) prior responses does not contribute to, and possibly interferes with, variable responding when an organism is reinforced for variability, and this, in turn, is consistent with the stochastic-generator hypothesis.

Stochastic response distributions. Other evidence is correlational: Response distributions are similar to those from a stochastic generator. The data from two experiments already discussed are shown in Figure 1. In these cases, reinforcement of least frequent IRTs (Blough, 1966) and of nonrepetitive choice sequences (Machado, 1989) resulted in stochastic-like responding.

Neuringer (1986) reported another striking case. High school and college students generated sequences of $1 \mathrm{~s}$ and $2 \mathrm{~s}$ on a computer keyboard, with each set of 100 responses constituting one trial. A baseline phase lasted for 60 such trials, for a total of 6,000 responses, during which the students were asked to behave as randomly as possible, as if they were reporting the outcome of a series of coin tosses. As in the many previous studies of this sort, the subjects' responses differed significantly from those of a stochastic model (Brugger, 1997; Wagenaar, 1972). During the training phase, the subjects received feedback, which enabled them to compare their performances with that of a stochastic model, first according to one statistical test and then according to another, until, following each trial, feedback was provided from 10 different statistics. The results were that all of the subjects learned to approximate the stochastic model according to the 10 statistics as well as some (but not all) other statistical tests. That is, the distributions of the statistics, which clearly did not match a stochastic model at the beginning of training, approximated the stochastic model at the end of it (see Figure 8).

The previous failures of other researchers to obtain evidence for random responding had led some to conclude that humans did not have the capacity to behave ran- domly. What would account for the difference between the present positive finding and the many other negative ones? Neuringer's (1986) study was the first in which stochastic responding was explicitly reinforced, in this case with feedback from 10 statistical tests. An analogy might help to explain the difference in procedures and results. Imagine an experimenter walking around a city and asking people if they know what a violin sounds like (analogous to asking people whether they know what a series of coin tosses or dice throws is like). Most would answer yes. If the same individuals were then asked to play the violin, very few, if any, would succeed. The experimenter might conclude, after many replications with different subjects in different cities, that people did not have the capacity to play the violin. The point, of course, is that playing the violin is a skill requiring training and practice, and the same may be true for responding randomly. It is easy to vary, but practice and reinforcement may be necessary to approximate a random distribution.

Response number. Page and Neuringer (1985) tested the stochastic-generator hypothesis in another way by varying number of responses per trial, with pigeons working under a lag 3 contingency. The reasoning was that if previous responses served as a discriminative cue for what not to do-a memory-based strategy - then performance should be degraded when the number of responses was increased: Eight responses per trial require subjects to remember more than four responses. The stochastic hypothesis predicts the opposite outcome, as is demonstrated by the following example. If each trial were two responses in length, with responses determined by the toss of a coin, then the probability that a given trial would repeat the previous one would be .25 . (There are four possible sequences in the first trial: RR, RL, LR, and LL. Thus, the second trial has a 1-in-4 chance of matching the first.) If a trial consists of four coin tosses, the probability of a repetition by chance is .0625 , or 1 in 16. Thus, if subjects used a stochastic process to generate Ls and Rs, performances should improve with increasing responses per trial. Results were precisely those predicted by the stochastic hypothesis and inconsistent with a memory strategy: Probability of meeting the contingencies, and therefore probability of reinforcement, increased as responses per trial increased. In other words, eight-response trials were easier for pigeons than four-response trials.

Response interference. Neuringer and Voss (2002) attempted to distinguish between memorial and stochastic processes in yet another way. They hypothesized that, whereas chaotic responding was based on memory for prior responses (as was discussed above), responding in Neuringer's (1986) study with feedback from 10 statistical tests indicated involvement of an endogenous stochastic generator. To show that human subjects could engage in both processes at will, three college students were trained to generate chaotic sequences in one component of a multiple schedule, as in Neuringer and Voss (1993), and to generate stochastic sequences in a sepa- 

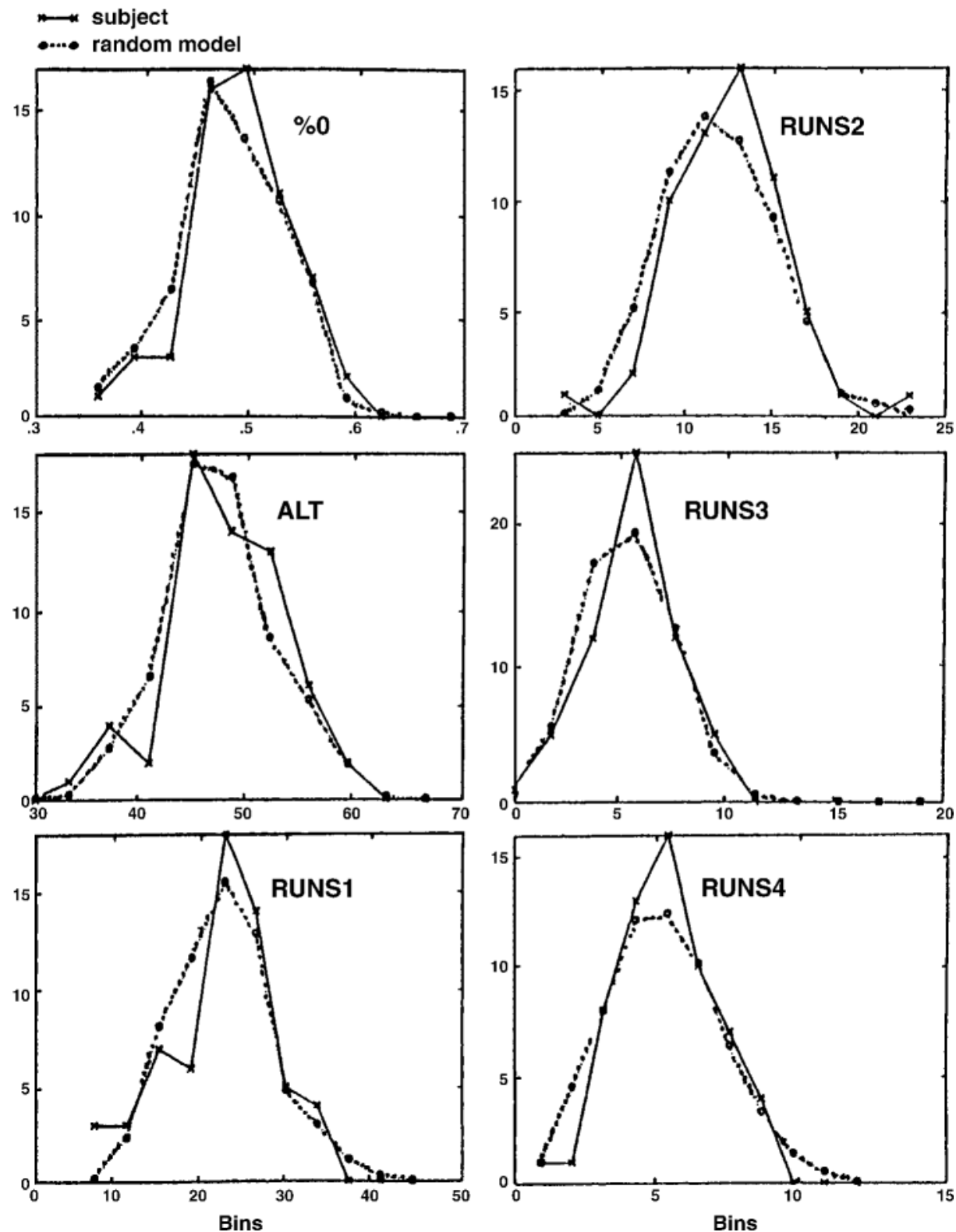

Figure 8. Performance by one subject at the end of training to respond stochastically in Neuringer (1986). Shown are comparisons on 6 different statistics between the subject (solid lines and Xs) and the random model (dashed lines and closed circles). At the end of training, the subject's performance did not differ statistically from that of the random model on these and four additional statistics. From “Can people behave 'randomly?': The role of feedback,' by A. Neuringer, 1986, Journal of Experimental Psychology: General, 115, p. 71. Copyright 1986 by the American Psychological Association. Reprinted with permission.

rate component, as in Neuringer (1986). The word CHAOTICS was on the screen in the first component, the word STOCHASTICS was on during the other, and these words alternated throughout the session. After the subjects succeeded at each of the component tasks, test sessions were introduced in which memory for prior responses was subject to interference in both the chaotics and stochastics components, as follows. During the chaotics component, four separate chaotic sequences were cycled one after the other, each cued by different screen colors. The subjects attempted to approximate a chaotic sequence in one color (e.g., light green), following which they would have to match a second chaotic sequence (lavender screen color), and so on, the four chaotic contingencies following one another across the session. Although all four chaotic sequences used the 
same logistic-difference equation that a model did, they were out of phase and independent. If chaotic responding is memory based, then the four independent sequences would be expected to interfere with one another; that is, approximations to the four chaotic models would be lower than when only a single chaotic output was required, as in Neuringer and Voss (1993). That was exactly the finding. Performances by the three subjects were significantly degraded during the four-segment phase. To put it simply, chaotic responses interfered with one another.

A different pattern of results was seen in the stochastics portion of the experiment. The same interference procedure was used, with four independentstochastic sequences required in the four different color conditions. As in Neuringer (1986), subjects were required to satisfy eight different statistical tests of randomness, and to do this independently in each of the four phases. (If responses in any two or more of the four stochastic phases became correlated above a minimal level, responses were considered to be incorrect, thereby prohibiting repetition of a single sequence in two or more of the phases.) For one subject, seven of eight statistics were closer to those of a random model during this interference phase than they were at the end of the original training with only a single sequence, and for a second subject, all eight statistics were closer. The third subject showed closer-to-random approximation under the four-segment condition on only two of the eight statistics, but most of her changes were also in the direction of a random generator. For example, if a random generator emits equal numbers of "up" and "down" sequences, and this subject in the baseline phase had emitted only $47 \%$ "up" sequences, then during the four-segment tests she came to emit "up" $55 \%$ of the time. She changed in the direction of the random model, but overshot the mark. Thus, whereas interference degraded chaotic performance significantly in all three subjects, it improved stochastic performances in at least two of them. These results are consistent with the hypothesis that a memory-based process controls chaotic variability, and that a stochastic process not dependent on memory controls stochastic variability.

\section{Summary of Tripartite Theory}

The following three processes constitute a tripartite theory of operant variability: attention to random events in the environment (coin toss); memorial processes (chaos, radial maze); and stochastic processes (response sequences and random-number generation with feedback). Random environmental events assure unpredictabilityfor example, in making unbiased selections. Remembering what was done previously will enable one to avoid repetitions: to vary the students called upon in class, to vary the locations when looking for a hidden object, or to try different approaches when attempting to solve a problem. External aids, such as notes to oneself or scientific records, help to enhance memory capacities.

An endogenous stochastic generator provides the third and most controversial source of behavioral variability.
When an opponent is attempting to predict your behavior, in a game or at war, it may be functional to behave stochastically. Similarly, operant conditioning of new responses may depend on stochastic variations. It is impossible to prove stochasticity; some presently unknown deterministic system may ultimately explain the results. However, the evidence is consistent with the stochastic hypothesis and inconsistent with the alternatives. For example, memory is not sufficiently powerful to explain all operant variability, memory-interference procedures leave stochastic responding intact, and behaviors correlate well with those of stochastic models.

Both genetics and reinforcement play important roles in all three processes. Reinforcement is always involved, in that learning provides information about when, what, where, and how much to vary. Genetic influences are shown, in that not all responses are possible, and if they are possible, they are not all equiprobable (e.g., as in Neuringer et al., 2001). Stochastic responding may be biased due to experiences and knowledge; that is, biases may be established by reinforcement contingencies or by the interface between the organism's propensities (or behavior systems; see Timberlake \& Lucas, 1989) and the environment. In the case of random-number generation by human subjects, biases may be due to misconceptions concerning what "random" means. However, some biases can be overcome through feedback and instructions (Lopes \& Oden, 1987; Neuringer, 1986).

The importance of variations for evolutionary change is supported by the many sources of genetic variability and by the fact that genetic variability is conserved. A similar argument holds for operant variability. That there are at least three independent sources of operant variations, as well as other elicitors such as environmental noise and response extinction, supports its importance for normal, adaptive functioning.

\section{Structure of Variability}

Every operant "response" is actually an instance of a class, with each member of the class exerting the same effect on the environment (Skinner, 1959). When a rat is reinforced for pressing a lever, for example, the class is defined by closure of a microswitch, and any way of accomplishing the closure, whether with the left paw, the right paw, or the mouth, is as effective in producing reinforcement as any other. If the contingencies of reinforcement so specify, however, the response class can be redefined and circumscribed, which is another characteristic of the operant. For example, if only right-paw presses are acceptable members of the reinforced class, responding will tend to occur only with the right paw (Stokes, 1995). Of course, the capabilities of the organism play a role; for example, including "tail presses" in the operant class does not mean that the lever would be, or could be, pressed by the rat's tail. The structure of the operant class, therefore, emerges from the current reinforcement contingencies and the organism's capability, derived from genetics, development, and prior experiences. 
Operant variability has structure as well and, as for all operants, that structure is due partly to the organism itself and partly to the reinforcement contingencies. The research described throughout this article has provided examples of the structure of variability, generally in the form of sets of four or eight responses across $L$ and $R$ operanda. But the structure of variations can be specified more precisely. For example, Mook and her colleagues (Mook et al., 1993; Mook \& Neuringer, 1994) reinforced rats for varying four response sequences across $\mathrm{L}$ and $\mathrm{R}$ levers under a lag 1 contingency. Unlike in previous studies, however, the acceptable class was further constrained so that only sequences beginning with two $\mathrm{R}$ responses (RRLL, RRLR, RRRL, and RRRR) were reinforced. The rats learned to vary their sequences within the imposed limitations; that is, they generally began their sequences with two $\mathrm{R}$ responses and varied the sequences within those limitations. Similar results were obtained in the context of a radial-arm maze in which only a subset of arms was baited.

The precision with which reinforcement contingencies can specify class was highlighted in research performed by Burns (1997) and Ross and Neuringer (2002). In the latter case, college students drew rectangles on a computer screen with the computer's mouse. Each rectangle constituted one trial (the screen cleared at the end of each trial), with reinforcement (points) depending on simultaneous variations along three dimensions of the rectangle: location on the screen, area of the rectangle, and shape, with the latter defined as the ratio of the rectangle's height to its width. Although the subjects were given no instruction other than to "gain points," they learned to vary along all three dimensions, with levels of variability significantly higher than those of yoked control subjects who were reinforced independently of variations.

A second part of the Ross and Neuringer (2002) study demonstrated even greater precision of control. To gain points, three new groups of subjects were required to repeat along one of the dimensions while simultaneously varying along the other two. One group was required to repeatedly draw rectangles of approximately the same size, while simultaneously varying location and shape; another group had to repeat the shape while varying area and location; and the third, to repeat location while varying size and shape. Verbal instructions were minimal, as they were in the first part of the study. Subjects learned to satisfy these contingencies as well, varying two attributes while simultaneously repeating one, and significant differences were obtained rapidly, within 300 trials.

These demonstrations raise questions as to how binary feedback-reinforcement presented or not-can simultaneously control independent behavioral dimensions. The issue is similar to that discussed above concerning simultaneous reinforcement of variations and selections. It is similar also to those issues faced by researchers attempting to understand how the nervous system strengthens particular synapses when many different neurons fire simultaneously and to "allocation of credit" in artificial learning models. For present purposes, suffice it to note the exquisite precision with which reinforcement controls variability and repetition.

\section{Generalization}

Do animals and people learn to "vary," implying a generalizable skill, or do they learn a specific distribution of specific responses across particular operanda? The question of generalization has been asked of many other behavioral competencies, such as imitation. When children learn to imitate, do they learn stimulus-response associations (if mother moves her right hand, the child learns to move his right hand), or do they learn more generally to "imitate?" Evidence indicates that reinforcement of imitative instances leads to generalized imitation (see, e.g., Baer \& Sherman, 1964; Bandura, 1986). A similar question has been raised when pigeons, rats, or children learn to choose a stimulus that matches a previously shown sample. For example, if a pigeon is shown a red disk as a sample, it must then choose red when given a choice between that and blue. Does the bird learn to match? In this case, the answer appears to depend on the training procedures. If trained with few stimuli, such as red, green, and blue, then the pigeon learns simple associations (if red sample, choose red; if blue sample, choose blue); but if hundreds of different stimuli (pictures) are employed during training, then generalized "matching" is learned (Wright, 1997). In each of these examples, the learning of a general competency, or strategy, was shown by transfer-of-training tests: What was learned with one set of stimuli or in one situation transferred to new stimuli and situations. We turn now to the question of whether learning to vary transfers.

After the subjects in Maltzman's (1960) experiment, described above, learned to generate remote verbal associates, they were asked to devise unusual uses of common objects. In comparison with those in the control group, these subjects generated significantly more unusual uses, indicating transfer of training to a different task. However, transfer was not observed in the opposite direction, from unusual uses to verbal associates.

Holman et al. (1977), using a procedure similar to that of the Goetz and Baer (1973) research described above, rewarded preschool children verbally for novel drawings and found increased novelty. Unrewarded block constructions were also monitored and weak evidence was obtained for transfer of novel responding from rewarded drawing to the unrewarded constructions. In a second experiment, preschoolers engaged in four independenttasks: painting, pen drawing, wood block construction, and Lego construction. When the children were reinforced for varying their pen drawings, the novelty of paintings increased along with the pen drawings, indicating transfer from one task to the other. However, transfer to the topographically different block- or Lego-building tasks was not observed.

Eisenberger and Armeli (1997) gave monetary rewards to one group of fifth- and sixth-grade students, the variability group, for describing unusual uses of common ob- 
jects. A second group, the repeat group, received the same rewards for describing common uses of the same objects. In a later test, all of the children were asked to draw pictures incorporating a circle, and the group that had previously been reinforced for varied responses drew more unusual (or lower frequency) pictures than did the repeat group. Thus, there was transfer of novelty training from one domain to another, with additional studies in the series showing similar effects (Eisenberger, Armeli, \& Pretz, 1998; Eisenberger, Haskins, \& Gambleton, 1999; Eisenberger \& Selbst, 1994). However, Eisenberger et al. (1999) failed to replicate the transfer-of-training effect: The variability and repeat groups responded similarly during the test phase. In this study, separate experimenters trained and tested the subjects, thereby avoiding possible experimenter-expectancy effects, whereas the same experimenter did both training and testing in Eisenberger's other studies. Across the series of experiments, there were many other cases in which transfer was not observed, and more research is necessary to explain why.

In Lee et. al's (in press) study with autistic individuals, described above, after variable answers were trained to questions, verbal variability transferred to new experimenters and new contexts.

Arnesen's (2000) results, described above, are consistent with transfer of training in an animal model. Rats in the Var group were trained to interact variably with one set of objects. When later tested in a new situation with new objects, the Var animals showed (in comparison with controls) higher levels of exploration, manipulation of the novel objects, and discovery of hidden reinforcers.

Much of the evidence reviewed in this section therefore indicates transfer of training and supports a general "vary" competency. However, there remain unresolved questions, the most important of which is the extent to which transfer occurs. Note also a methodological point. Many of the studies tested whether transfer occurs when the contingencies are neutral with respect to the to-betransferred behavior. Holman et al. (1977) is a case in point: Variations were reinforced in one domain, drawing, and the question was whether variations would occur in another domain - block construction-in which reinforcement did not depend on varying. A negative finding - in this case, absence of transfer-indicates nothing about the ability to generalize, but only about the tendency to do so given the absence of a reason (or contingency). A better test would be to compare experimental and control performances when variability in the new task is, in fact, functional, as in Arnesen (2000).

\section{Summary}

Before variability can be reinforced, it must occur for reasons other than reinforcement. Some of the reasons include variability inherent in all behavioral phenomena, "accidental" environmental events that are unrelated to particular behaviors, and withholding of reinforcement. Transfer from other situations may serve a similar function. For example, if an organism can discriminate shaping- of-new-response periods from others, then it may learn generally to "vary" during shaping episodes. After reinforcement makes initial contact with response variations, there are at least three behavioral strategies that can be employed in order to maintain functionally high levels of variability: respond based on external random events, such as the toss of a coin; avoid repetitions by remembering what responses had previously been made; and respond stochastically. Evidence for an endogenous stochastic process was seen in the similarity of response distributions to those generated by stochastic sources, and by findings that interfering with memory for prior responses either improves or leaves unaffected stochastic behaviors. Stochastic responding, as well as other types of variability-generating strategies, depend upon contingencies to define the required level of variability and the set from which variations emerge.

\section{IMPLICATIONS}

One implication of reinforced variability is that operant responses may differ in the extent to which their variability is affected by reinforcement contingencies-that is, operants may be ordered along a continuum of sensitivity of variability to reinforcement. Another is that reinforced variability causes us to question the generality of some basic goals of behavioral psychology, including prediction and control. A third is that reinforced variability may help us to provide a scientifically useful way to describe voluntary action. In particular, the variability of all voluntary behaviors can readily be changed, moment to moment, by discriminative stimuli and consequences. Implications for everyday life also will be noted.

\section{Operant Continuum}

As was indicated above, the operant is composed of instances; that is, the operant is a class of many different responses, all functionally equivalent but often topographically distinct. Skinner suggested, and I hypothesize, that instances within the class emerge stochastically (see Moxley, 1997, for a discussion of Skinner's position). Although reinforcement contingencies and context influence the individual probabilities and their distributions, emission is stochastic nonetheless: The particular member of the operant class generally cannot be predicted with a high degree of certainty. For example, a particular rat may be more likely to press the lever with its left paw than its right, but left- and right-paw responses are emitted probabilistically. This class-nature of the operant suggests one way to conceptualize operant variability: in terms of size of class and within-classes probability distributions. High variability implies large classes, equal probability distributions, or both; low variability implies the opposite.

Variability may be modified more readily in some operants than in others, implying that operant variability is not an all-or-none phenomenon, but rather a continuum, with reinforced variations in variability (RVV) differing 
for different operants. RVV might best be understood by analogy to the concepts of speed and acceleration. Both variability and speed range from low to high. RVV is analogous to acceleration, the rate of change in speed. Highly sensitive RVV is indicated by the possibility of reinforcing many different levels of variability, from repetition to random-like responding, and by the fact that changes in level occur readily and rapidly.

Different operants manifest differences in RVV. For example, variability of chain pulls changed more than that of leverpresses when variability was reinforced in rats (Morgan \& Neuringer, 1990). Some operant-like responses may show relatively low RVV. For example, firing rates increase in single hippocampal nerve cells when dopaminergic substances are contingent upon the response (Stein, Xue, \& Belluzzi, 1994), suggesting that nerve firing is controlled by reinforcement. To date, however, there is no evidence that variability of nerve firing is similarly controlled. Other cases might include reinforcement of nondiscriminable (subconscious) responses, such as Hefferline and Perera's (1963) reinforcement of subaware thumb twitches, and biofeedback of muscle and cardiovascular activity. In these cases, reinforcement may modify performance, but it is not known whether variability is also sensitive to reinforcement and, if so, to what degree. Human verbal variability may be the most readily manipulable of all behaviors, the range of predictable to unpredictable verbal responses being immense, with extraordinary sensitivity to context and consequence. RVV may also differ across organisms (perhaps from simple to complex), individuals (perhaps as an index of intelligence or learning ability), and physiological and psychological states (WKY vs. SHR, depression, and autism).

RVV may help to distinguish operant responses from other superficially similar cases of selection by consequences. That is, the "operant" may also best be conceptualized as a continuum, with RVV an important determiner of position along the operant continuum. One example of selection by consequences but low "operantness" is genetic change. Variability is essential for evolutionary change, of course, and levels of genetic variability are sometimes affected by environmental conditions (Moxon, 1997), but there are few examples of levels of variability being influenced by selection pressures. That is, the source of much genetic variability is thought to be random, not influenced by current pressures and needs. Thus, although selected by consequences, genetic change may be low along the operant continuum. Other examples of operant-like phenomena for which variability, although important, may be relatively insensitive to consequences-variability in these cases is elicited, not selected-include the development of the central nervous system and immune system responses (Hull et al., 2001). In both of these cases, change depends upon selection by consequences, but it is not clear whether variability can also be selected. Most commonly studied operant responses, on the other hand, show high sensitivity of vari- ability to reinforcing events. But, to reiterate the main point of this section, operants differ in terms of RVV and therefore in terms of their "operantness," from low sensitivity (possibly responses of single nerve cells) to extraordinarily high sensitivity (verbal behavior). The operant nature of variability, as well as that of every other operant dimension, is best conceptualized as a continuum.

\section{Prediction and Control}

Smith (1992) described Skinnerian operant conditioning theory as Baconian in nature, a primary goal of Skinnerian behaviorists being to control behavior, and he contrasted this with the more Aristotelian goal of description. Skinner and his students (e.g., Zuriff, 1985) indeed emphasized prediction and control. Many of the studies reviewed above, however, involved reinforcement procedures that increase variability and consequently decrease predictability. It is not only ignorance, therefore, that restricts prediction and control, as Skinner suggested, but reinforcement itself. Operant methods and theories, when applied to variability, lead to greater emphasis on description, with control over precise response characteristics possible under some, but not all, conditions.

In summary, an operant is a class of responses under the control of contingencies of reinforcement. The class can be controlled and predicted, given knowledge of conditioning history. Indeed, the size of the class and possibly the distribution of within-classes probabilities, or level of variability, can also be controlled and predicted. But, to the extent that instances emerge stochastically, a particular instance cannot be predicted. The instant response can be explained as a member of a particular class, but prediction of the instance will be difficult, sometimes to the extreme. This point will be developed in the next section.

\section{Voluntary Behavior}

I hypothesize that voluntary behaviors are operants for which RVVs are highly sensitive to influence by consequences. One way to test this hypothesis would be to gather empirical data concerning how voluntary versus involuntary actions are classified by human observers. As far as I know, there are no psychophysical data concerning this question, but the hypothesis implies that high sensitivity of variability to operant contingencies, RVVs, will be present in all actions perceived to be voluntary.

This operant-variability theory of voluntary action is based on the supposition of two attributes. First, voluntary acts must be potentially explainable (given sufficient knowledge) on the basis of genetics, conditioning histories, current contingencies, anticipated consequences, intentions, goals, and the like. That is, voluntary behaviors are lawful. The second attribute is that the voluntary act must be potentially unpredictable. That is, voluntary acts are "free" of control by identifiable stimuli. If behavior is perceived necessarily to result fromto be predicted on the basis of-external events, includ- 
ing reinforcement contingencies, it will not be perceived as voluntary. "Voluntary" implies potential independence from stimulus-response or response-reinforcer determination. According to this theory, both explainability and unpredictability are necessary attributes of voluntary acts.

How, though, can behaviors be simultaneously explainable and unpredictable? Reinforcement contingencies play two essential roles. Reinforcement (together with genes, prior experiences, and current environment) helps to determine the set of possible behaviors from which a voluntary act emerges - that is, the operant class. Reinforcement (together with the other contributors specified above) also helps to determine the level of variability within the class. Thus, both instances comprising a class and level of variability within that class can be predicted-again, given sufficient knowledge. However, the individual response is at least sometimes (or relatively) unpredictable. The reason is that the operant class from which the response stochastically emerges is sometimes extremely large (e.g., "name a word," or "do something"), and the contingencies sometimes call for equiprobable, stochastic emergence (e.g., "fool me"). In the latter case, a voluntary act may appear to be foolish, meaningless, or unwarranted, but if it is voluntary, it can potentially be explained. (For example, in response to the question: "Why did you do that silly act?" the answer could be: "Just to show that you can't predict my behavior"; see Scriven, 1965.) In these cases, prediction of instances falls to very low levels indeed, and, I hypothesize, even an all-knowing observer will be unable to predict the next response at greater-than-chance levels, which are given by the size of the operant class and by within-classes probability distribution. Even within small classes (e.g., in the game rock-scissors-paper, in which unpredictability among the three possible responses is functional), behavioral stochasticity may lead to unpredictability of the instant response. Note, however, that although voluntary behaviors are sometimes unpredictable, the opposite is often the case. When contingencies call for highly constrained, particular responses, predictions can easily be made. For example, if a police officer asks your name, your response can most likely be predicted. Voluntary responses are therefore described as potentially unpredictable, with variability contingencies engendering different levels of unpredictability.

According to this theory, behaviors low in the sensitivityof-variability dimension are described as relatively involuntary, and those that are high in said dimension are perceived as voluntary. Low RVV may be shown at any level along the variability continuum. For example, always responding in the same, unchanging way will be perceived as involuntary, but so will always responding randomly. (The coin does not voluntarily come up with heads or tails.) This point is essential. More than 2,000 years ago, Epicurus argued that occasional random swerves of atoms provided the source of voluntary behavior in an otherwise determined universe, but, al- though this conjecture was prescient, it was not quite correct. "Free-willed" or voluntary action, whether it is based on random swerves or on quantum mechanics, is not explained by variability per se but by functional changes in levels of variability - that is, by rapid and precise changes in response to environmental demands. Responses can be placed along a voluntary-involuntary continuum, isomorphic to the operant-respondent continuum, with voluntary behavior being functional precisely in the same way as operant behavior is. Thus, Skinner's (1974) suggestion that voluntary behavior is operant behavior was almost correct: Only those operants for which variability is highly sensitive to consequences will be perceived as voluntary.

One additional example might help. Operant classes (sets of potential responses) are created "on line" as we go about our daily activities. As we walk from car to office, we tend to walk in a characteristic way, take the same path, with tightly constrained operant classes following one after the other in rapid succession. Class sizes are small, and within-classes probability distributions are highly peaked. Predictions of next responses by a knowledgeable observer can readily be made. However, class sizes can be increased and probability distributions flattened; we can walk in quite strange ways, indeed dance along a new path, sing, hand candy to passers-by. Strange behavior, indeed, but made possible by the ability of operant sets to be modified moment to moment, not only in terms of class definition, but in terms of class size, levels of within-classes variations, and so on. The same is true for conversation: Moment-to-moment changes in verbal class definition, size, and predictability of withinclasses instances are hallmarks of voluntary behavior.

\section{Generativity}

Related to the definition of the operant and to our discussion of voluntary action is that fact that operant behaviorleverpressing no less than language - is, by its nature, generative. This claim follows from the functional nature of the class of responses (all members of the class are effective in producing an outcome) and the stochastic emission of instances. From these flow never-beforeemitted instances, patterns, and sequences (see Epstein, 1996). Operants, especially those high on the RVV continuum, are inherently generative.

\section{Self-Experimentation}

Variations, systematic or otherwise, are a necessary part of scientific work. Neuringer (1981) and Roberts and Neuringer (1998) described how variations can be brought to bear explicitly on everyday life in the form of selfexperiments. Individuals vary aspects of their lives, such as the foods they eat, social contacts, and methods of memorizing; they keep records concerning the effects of these manipulations and form hypotheses concerning controlling variables. Using self-experimental methods, individuals can learn how, what, when, where, and how much to vary. Explicitly varying one's everyday behav- 
iors and thoughts may help to engender what Langer $(1989,2000)$ has referred to as mindful living - that is, nonautomatic, voluntary engagement, with beneficial effects in different areas, including learning. The important point here is that variability can be voluntarily chosen, maintained, and modified in everyday life, and that explicit variations may have important consequences.

\section{Implications for Society}

Consideration should be given to the importance of reinforcement contingencies to support variability in social milieus. A basic point made throughout the present article is that merely permitting behavior to vary does not necessarily engender or maximize variability (as is shown by Yoke and Prob schedules). Analogously, merely permitting varied social behavior, as in a free or laissezfaire society, may not suffice to produce the desired diversity. If the results, shown for individual organisms, can provide useful information for groups (see, e.g., Grott $\&$ Neuringer, 1974), then when variations are desired, societies might reward individuals for varying, not simply permit it.

\section{Summary}

Operants differ in the extent to which reinforcers influence levels of variation. When variability is highly sensitive to reinforcement, the operant may be perceived to be voluntary. Voluntary responses have two attributes. They are functional, implying that the class of responsesthe operant - can be predicted, given sufficient knowledge. But instances emerge stochastically, implying some degree of unpredictability. Because reinforcement of variability can generate extremely large classes, with stochastic emergence of instances, it suggests limitations of the two basic goals of Skinnerian behavioral theorynamely, prediction and control. Operant variability also has implications for self-experimentation and for society.

\section{RELATED AREAS}

Operant variability may be important in many other areas, and I will briefly review some of these.

Negative reinforcement. To my knowledge, there have been no published studies in which variability is negatively reinforced. High variability might sometimes avoid or reduce a punisher, such as loss of goods or money, but at other times, low variability could be functional. Effects of positive and negative reinforcement on variability may have different evolutionary bases, one derived from increasing probabilities of locating sources of food or mates, the other from avoiding confrontations or predation (Driver \& Humphries, 1988). One question concerns how quickly variability can be changed by negative, as opposed to positive, reinforcement. There is considerable evidence that loss functions differ from gain functions (Kahneman \& Tversky, 1984), possibly indicating that positive reinforcement-of-variability functions will differ from negative ones as well.
Resistance to change. In some of the studies described above, variability appeared to be more resistant to change than repetitions were; for example, alcohol had little effect on variable operant responses but interfered with repeated ones. Doughty and Lattal (2001) tested the generality of these findings using four-response sequences with pigeons, a threshold variability contingency in the Var component of a multiple schedule, and LRLR in the Rep component. Doughty and Lattal challenged stability of responding by prefeeding the birds in one experiment, and providing reinforcers independently of responding in the other (see Nevin, 1992). Operant variability was again more resistant to change than was operant repetition. Thus, reinforcement of variability might increase the stability of responding (that is, its resistance to change), a seemingly paradoxical finding. However, Mechner (1992) suggested that this is the case for complex skills such as piano performance: Varying practice routines will make the performance relatively insensitive to unforeseen changes in the environment. Care must be taken, however, when variation is compared with repetition, because they have different measures. Also, the results reported by Doughty and Lattal were based on the performance of three pigeons and were not consistent throughout the experiment. The hypothesis warrants further study.

Attraction. In some bird species, complex male songs attract females (for a review, see Catchpole \& Slater, 1995). There is evidence that male song complexity is under the discriminative stimulus control of a receptive female (Searcy \& Yasukawa, 1990). That explicit reinforcement may play a role in such song complexity is suggested by the Manabe et al. (1997) study, described above. An interesting question for further research is the extent to which operant contingencies contribute to the attraction created by behavioral variability, in these and other species.

Discrimination of variability. Animals and people can use the variability of a stimulus array, or entropy, as a discriminative stimulus (Young \& Wasserman, 2001). What is the relationship between perception of variability, on the one hand, and emission of variable responses, on the other? Do individuals attempt to match their own levels of variation to those of others in their environments, which would involve both perception and emission? How might discrimination of one's own level of variability influence one's ability to modify such levels, and do influences that obscure discriminations therefore affect ability to change (see, e.g., van der Meer, van der Weel, \& Lee, 1995)? Hunziker et al. (2002) report that, although variability was successfully reinforced, their human subjects could not correctly describe the contingencies of reinforcement, a finding similar to those of informal observations in other studies (e.g., Neuringer, Deiss, \& Imig, 2000), but whether subjects could discriminate variability levels rather than contingencies was not studied.

Attention and awareness. When attention is subject to interference or is withdrawn from the task at hand, ability to vary is degraded (e.g., Baddeley, Emslie, Kolodny, \& 
Duncan, 1998; Evans \& Graham, 1980; Neuringer \& Voss, 2002). It is unclear at present how attention influences the different processes underlying operant variability, and in particular, memorial and stochastic processes. Rich areas for additional study are the relationships between attention, awareness, and consciousness, on the one hand, and operant variability, on the other. Voluntary action and consciousness are often related (see, e.g., Libet, 1999).

Concepts. Concepts are thought by many to be sets of instances (much like operant sets) whose structures are graded, meaning that there are more or less typical instances of such concepts as birds (robin is typical, ostrich is not). Barsalou (1987) showed that the gradation of these cognitive structures is manipulable, or, as he puts it, not stable. A number of variables influence structure, including context, individual differences, and points of view. Barsalou argued that these structures are created as the need arises - that is, on line by the thinking individual. The structure of concepts may parallel that of operants, and in both cases, levels of within-structures diversity or variability may be modified by consequences. Operant variability may be found in the cognitive as well as in the behavioral domain.

Play and humor. Highly variable behaviors are observed when animals and people, especially children, engage in play and humor (see, e.g., Fagen, 1981). The result of play may be acquisition of new responses (see Sutton-Smith, 1975). Although not currently needed, responses learned during play may provide the source of variations for successful shaping of behaviors at another time. This is quite analogous to the role of conserved genetic variants, which are passed along to progeny but do not currently serve any identifiable role. Play may also involve learning how to vary operantly.

Reinforcement by variability. The self-maintaining nature of play suggests that engaging in variable behaviors may itself be reinforcing. Indirect support comes from findings that varied stimulation can serve as a reinforcer and that stimulus repetition becomes aversive (Fiske \& Maddi, 1961). Possibly also related are findings that varying reinforcers and permitting choice among reinforcers increase their value (Bowman, Piazza, Fisher, Hagopian, \& Kogan, 1997), and that permitting choices among varied responses is also preferred to demanding fixed responses (Catania, 1980).

Foraging. Two issues regarding variability are present in the foraging literature. One has to do with risk preference versus avoidance (see, e.g., Shettleworth, 1998). A second has to do with search strategies. Most reported cases of foraging involve systematic search patterns (Stephens \& Krebs, 1986). However, search patterns are sometimes stochastic, or highly variable (see, e.g., Peterson, 1997). One question is whether some species can vary between these two strategies, and the challenge is to identify the pressures leading to, and the advantages of, each.

Animal training. Wilkes (1996) has applied operant variability techniques in developing training procedures for pet dogs. More generally, reinforcement of variabil- ity may enable the gaining of control over behaviors of animals and, as has been suggested above, may be an important part of the process of shaping.

Models of learning. Reinforced variations in variability may be important when human and animal learning is being modeled, whether the models are mathematical in form, computer based, or machine based (Blumberg et al., 2002; Brooks, 1999; Sutton \& Barto, 1998).

\section{CONCLUDING COMMENT}

Genes interact with environments to produce diverse anatomical, physiological, and behavioral phenotypes. Genes and environments also provide for another level of diversity - namely, operant variability. There are many sources of operant variability, including random environmental events, memory for past responses, and stochastic generation, each of which ultimately derives from genetic and environmental influences. To the extent that operant variability is based on a stochastic source, however, the behavioral results cannot be predicted from knowledge of these influences. That is, organisms have evolved to behave unpredictably. The positing of stochasticity to explain behavior has a history extending at least as far back as Epicurus, with major contributors including Gustav Fechner (see Heidelberger, 1987), Egon Brunswik (see Gigerenzer, 1987), Skinner (1959), and Suppes (1984). Knowledge of the stochastic nature of the operant and of how it is controlled leads to a world view that differs from genetic and environmental determinism, with roots in the works of the scholars just mentioned. At the scientific level, this view leads to the experimental analysis of consequence-influenced variability. At the philosophical level, it leads to explanations of voluntary action. At the personal level, it can lead to change.

\section{REFERENCES}

Amabile, T. M. (1983). The social psychology of creativity. New York: Springer-Verlag.

Antonitis, J. J. (1951). Response variability in the white rat during conditioning, extinction, and reconditioning. Journal of Experimental Psychology, 42, 273-281.

Archer, J., \& Birke, L. (1983). Exploration in animals and humans. Cambridge: Van Nostrand Reinhold.

Arnesen, E. M. (2000). Reinforcement of object manipulation increases discovery. Unpublished undergraduate thesis, Reed College.

BADDELEY, A. D. (1966). The capacity for generating information by randomization. Quarterly Journal of Experimental Psychology, 18, 119-129.

Baddeley, A. [D.], Emslie, H., Kolodny, J., \& Duncan, J. (1998). Random generation and the executive control of working memory. Quarterly Journal of Experimental Psychology, 51A, 819-852.

BAER, D. M., \& Sherman, J. A. (1964). Reinforcement control of generalized imitation in young children. Journal of Experimental Child Psychology, 1, 37-49.

BAKer, M. J., \& Koegel, R. L. (1999). Transforming children with autism's ritualistic behaviors into positive play interactions with siblings. Paper presented at the meeting of the California Association of Behavior Analysis, San Francisco.

Balsam, P. D., Deich, J. D., Ohy ama, T., \& Stokes, P. D. (1998). Origins of new behavior. In W. O'Donohue (Ed.), Learning and behavior therapy (pp. 403-420). Boston: Allyn \& Bacon.

Balsam, P. D., Paterniti, A., Zechowy, K., \& Stokes, P. D. (2002). 
Outcomes and behavioral variability: Disappointment induced variation. Manuscript submitted for publication.

Balsam, P. D., \& Silver, R. (1994). Behavioral change as a result of experience: Toward principles of learning and development. In J. A. Hogan \& J. J. Bolhuis (Eds.), Causal mechanisms of behavioural development (pp. 327-357). Cambridge: Cambridge University Press.

BAndura, A. (1982). The psychology of chance encounters and life paths. American Psychologist, 37, 747-755.

BANDURA, A. (1986). Social foundations of thought \& action. Englewood Cliffs, NJ: Prentice-Hall.

Barba, L. S., \& Hunziker, M. H. (2002). Variabilidade comportamental produzida por dois esquemas de reforçamento (Behavioral variability produced by two reinforcement schedules). Acta Comportamentalia, 10, 5-22.

BARKLEY, R. (1990). Attention deficit hyperactivity disorder: A handbookfor diagnosis and treatment. New York: Guilford.

BARon-Cohen, S. (1992). Out of sight or out of mind? Another look at deception in autism. Journal of Child Psychology \& Psychiatry, 33, 1141-1155.

BARSALOU, L. W. (1987). The instability of graded structure: Implications for the nature of concepts. In U. Neisser (Ed.), Concepts and conceptual development: Ecological and intellectual factors in categorization (pp. 101-140). New York: Cambridge University Press.

BECK, A. T. (1976). Cognitive therapy and the emotional disorders. New York: International Universities Press.

Berryman, R., Cumming, W. W., \& Nevin, J. A. (1963). Acquisition of delayed matching in the pigeon. Journal of the Experimental Analysis of Behavior, 6, 101-107.

Beveridge, W. I. B. (1957). The art of scientific investigation. New York: Vintage.

Blough, D. S. (1966). The reinforcement of least frequent interresponse times. Journal of the Experimental Analysis of Behavior, 9, 581-591.

Blumberg, B., Downie, M., Ivanov, Y., Berlin, M., Johnson, M. P., \& TomLinson, B. (2002). Integrated learning for interactive synthetic characters. Transactions on Graphics 21, 3 (Proceedings of ACM SIGGRAPH 2002).

Boden, M. A. (1994). What is creativity? In M. A. Boden (Ed.), Dimensions of creativity (pp. 75-117). Cambridge, MA: MIT Press.

Boren, J. J., Moerschbaecher, J. M., \& Whyte, A. A. (1978). Variability of response location on fixed-ratio and fixed-interval schedules of reinforcement. Journal of the Experimental Analysis of Behavior, 30, 65-67.

Bouton, M. (1994). Context, ambiguity, and classical conditioning. Current Directions in Psychological Science, 3, 49-53.

Bowman, L. G., Piazza, C. C., Fisher, W. W., Hagopian, L. P., \& Kogan, J. S. (1997). Assessment of preference for varied versus constant reinforcers. Journal of Applied Behavior Analysis, 30, 451-458.

Breland, K., \& Breland, M. (1961). The misbehavior of organisms. American Psychologist, 16, 681-684.

Brooks, R. A. (1999). Cambrian intelligence: The early history of the new AI. Cambridge, MA: MIT Press.

Brugger, P. (1997). Variables that influence the generation of random sequences: An update. Perceptual \& Motor Skills, 84, 627-661.

Bryant, D., \& Church, R. M. G. (1974). The determinants of random choice. Animal Learning \& Behavior, 2, 245-248.

BuRns, J. (1997). Training changing triangles: A new paradigm for examining behavioral variability in multiple dimensions. Unpublished undergraduate thesis, Reed College.

Cameron, J., \& Pierce, W. D. (1994). Reinforcement, reward, and intrinsic motivation: A meta-analysis. Review of Educational Research, 64, 363-423.

CAMPBELl, D. T. (1960). Blind variation and selective retention in creative thought as in other knowledge processes. Psychological Review, 67, 380-400.

Carlton, P. L. (1962). Effects of deprivation and reinforcementmagnitude on response variability. Journal of the Experimental Analysis of Behavior, 5, 481-486.

Catania, A. C. (1980). Freedom of choice: A behavioral analysis. In G. H. Bower (Ed.), The psychology of learning and motivation (Vol. 14, pp. 97-145). New York: Academic Press.
Catchpole, C. K., \& Slater, P. J. (1995). Bird song: Biologicalthemes and variations. Cambridge: Cambridge University Press.

Channon, S., \& Baker, J. E. (1996). Depression and problem-solving performance on a fault-diagnosis task. Applied Cognitive Psychology, 10, 327-336.

Cherot, C., Jones, A., \& Neuringer, A. (1996). Reinforced variability decreases with approach to reinforcers. Journal of Experimental Psychology: Animal Behavior Processes, 22, 497-508.

Cohen, L., Neuringer, A., \& Rhodes, D. (1990). Effects of ethanol on reinforced variations and repetitions by rats under a multiple schedule. Journal of the Experimental Analysis of Behavior, 54, 1-12.

Cumming, W. W., Berryman, R., Cohen, L. R., \& Lanson, R. N. (1967). Some observations on extinction of a complex discriminated operant. Psychological Reports, 20, 1328-1330.

Deci, E. L., Koestner, R., \& Ryan, R. M. (1999). A meta-analytic review of experiments examining the effects of extrinsic rewards on intrinsic motivation. Psychological Bulletin, 125, 627-668.

Dember, W. N., \& Richman, C. L. (1989). Spontaneous alternation behavior. New York: Springer-Verlag.

DenNey, J., \& Neuringer, A. (1998). Behavioral variability is controlled by discriminative stimuli. Animal Learning \& Behavior, 26, 154-162.

DeVEnPoRT, L. D. (1983). Spontaneous behavior: Inferences from neuroscience. In R. Mellgren (Ed.), Animal cognition and behavior (pp. 83125). Amsterdam: North-Holland.

Doughty, A. H., \& LatTal, K. A. (2001). Resistance to change of operant variation and repetition. Journal of the Experimental Analysis of Behavior, 76, 195-215.

Dover, A., \& Shore, B. M. (1991). Giftedness and flexibility on a mathematical set-breaking task. Gifted Child Quarterly, 35, 99-105.

Driver, P. M., \& Humphries, D. A. (1988). Protean behavior: The biology of unpredictability. Oxford: Oxford University Press.

Duncan, C. P. (1961). Attempts to influence performance on an insight problem. Psychological Reports, 9, 35-42.

Eckerman, D. A., \& LANson, R. N. (1969). Variability of response location for pigeons responding under continuous reinforcement, intermittent reinforcement and extinction. Journal of the Experimental Analysis of Behavior, 12, 73-80.

Eisenberger, R., \& Armeli, S. (1997). Can salient reward increase creative performance without reducing intrinsic creative interest? Journal of Personality \& Social Psychology, 72, 652-663.

Eisenberger, R., Armeli, S., \& Pretz, J. (1998). Can the promise of reward increase creativity? Journal of Personality \& Social Psychology, 74, 704-714.

Eisenberger, R, \& CAMERon, J. (1996). Detrimental effects of reward: Reality or myth? American Psychologist, 51, 1153-1166.

Eisenberger, R., Haskins, F., \& Gambleton, P. (1999). Promised reward and creativity: Effects of prior experience. Journal of Experimental Social Psychology, 35, 308-325.

Eisenberger, R, \& Selbst, M. (1994). Does reward increase or decrease creativity? Journal of Personality \& Social Psychology, 66, 1116-1127.

EPSTEIN, R. (1996). Cognition, creativity and behavior: Selected essays. Westport, CT: Praeger.

Evans, F. J., \& GRAHAM, C. (1980). Subjective random number generation and attention deployment during acquisition and overlearning of a motor skill. Bulletin of the Psychonomic Society, 15, 391-394.

FAGEN, R. (1981). Animal playbehavior. New York: Oxford University Press.

Ferster, C. B., \& Skinner, B. F. (1957). Schedules of reinforcement. New York: Appleton-Century-Crofts.

FISKE, D. W., \& MADDI, S. R. (1961). Functions of varied experience. Homewood, IL: Dorsey.

GALBICKA, G. (1994). Shaping in the 21st century: Moving percentile schedules into applied settings. Journal of Applied Behavior Analysis, 27, 739-760.

Gigerenzer, G. (1987). Survival of the fittest probabilist: Brunswik, Thurstone, and the two disciplines of psychology. In L. Kruger, G. Gigerenzer, \& M. S. Morgan (Eds.), The probabilistic revolution. Vol. 2: Ideas in the sciences (pp. 49-72). Cambridge, MA: MIT Press. Goetz, E. M., \& BAER, D. M. (1973). Social control of form diversity 
and emergence of new forms in children's blockbuilding. Journal of Applied Behavior Analysis, 6, 209-217.

GoRKA, E. (1993). Within session effects of ethanol on reinforced behavioral variability. Unpublished undergraduate thesis, Reed College.

Gott Lieb, G. (1992). Individual development and evolution. Oxford: Oxford University Press.

Grott, R., \& Neuringer, A. (1974). Group behavior of rats under schedules of reinforcement. Journal of the Experimental Analysis of Behavior, 22, 311-321.

Grunow, A., \& Neuringer, A. (2002). Learning to vary and varying to learn. Psychonomic Bulletin \& Review, 9, 250-258.

Guthrie, E. R, \& Horton, G. P. (1946). Cats in a puzzle box. New York: Rinehart.

Hefferline, R. F., \& Perera, T. B. (1963). Proprioceptive discrimination of a covert operant without its observation by the subject. Science, 139, 834-835.

HeIDELBERGER, M. (1987). Fechner's indeterminism: From freedom to laws of chance. In L. Kruger, L. J. Daston, \& M. Heidelberger (Eds.), The probabilistic revolution. Vol. 1: Ideas in history (pp. 117-156). Cambridge, MA: MIT Press.

HerRnsteIn, R. J. (1961). Stereotypy and intermittent reinforcement. Science, 133, 2067-2069.

Hertzig, M. E., \& Shapiro, T. (1990). Autism and pervasive developmental disorders. In M. Lewis \& S. M. Miller (Eds.), Handbook of developmental psychopathology (pp. 385-395). New York: Plenum.

Holman, J., Goetz, E. M., \& BaER, D. M. (1977). The training of creativity as an operant and an examination of its generalization characteristics. In B. Etzel, J. LeBland, \& D. Baer (Eds.), New developments in behavior research: Theory, method and application (pp. 441-471). Hillsdale, NJ: Erlbaum.

Hopkinson, J., \& NEURINGER, A. (in press). Modifying behavioral variability in moderately depressed students. Behavior Modification.

Hopson, J., Burt, D., \& Neuringer, A. (2002). Variability and repetition under a multiple schedule. Manuscript in preparation.

Horne, R. L., Evans, F. J., \& Orne, M. T. (1982). Random number generation, psychopathology, and therapeutic change. Archive of General Psychiatry, 39, 680-683.

Hull, D. L., Langman, R. E., \& Glenn, S. S. (2001). A general account of selection: Biology, immunology and behavior. Behavioral \& Brain Sciences, 24, 511-573.

Hunziker, M. H. L., Caramori, F. C., Da Silva, A. P., \& Barba, L. S. (1998). Efeitos da historia de reforçamento sobre a variabilidade comportamental [Effects of reinforcement history on behavioral variability]. Psicologia: Teoria e Pesquisa, 14, 149-159.

Hunziker, M. H. L., Lee, V. P Q., Ferreira, C. C., DA Silva, A. P., \& CARAMORI, F. C. (2002). Variabilidade comportamental em humanos: Efeitos de regras e contingencias [Human behavioral variability: Effects of rules and contingencies]. Psicologia: Teoria e Pesquisa, 18, 130-147.

Hunziker, M. H. L., Saldana, R. L., \& Neuringer, A. (1996). Behavioral variability in SHR and WKY rats as a function of rearing environment and reinforcement contingency. Journal of the Experimental Analysis of Behavior, 65, 129-144.

Kahneman, D., \& Tversky, A. (1984). Choices, values and frames. American Psychologist, 39, 341-350.

KIlleEn, P. (1981). Incentive theory. In D. J. Bernstein (Ed.), Nebraska symposium on motivation, 1981: Response structure and organization (pp. 169-216). Lincoln: University of Nebraska Press.

KNUTH, D. E. (1969). The art of computer programming. Reading, MA: Addison-Wesley.

LAmberty, Y., \& Gower, A. J. (1990). Age-related changes in spontaneous behavior and learning in NMRI mice from maturity to middle age. Physiology \& Behavior, 47, 1137-1144.

LANGer, E. (1989). Mindfulness. Reading, MA: Addison-Wesley.

Langer, E. (2000). Mindful learning. Current Directions in Psychological Science, 6, 220-223.

LAPP, J. E. MArinier, R., \& Pihl, R. O. (1982). Correlates of psychotropic drug use in women: Interpersonal personal problem solving and depression. Women \& Health, 7, 5-16.

Lee, R, McComas, J. J., \& JaWor, J. (in press). The effects of differential reinforcement on varied verbal responding by individuals with autism to social questions. Journal of Applied Behavioral Analysis.
Lepper, M. R., \& Henderlong, J. (2000). Turning "play” into "work" and "work" into "play": 25 years of research on intrinsic versus extrinsic motivation. In C. Sansone \& J. M. Harackiewicz (Eds.), Intrinsic and extrinsic motivation: The search for optimal motivation and performance (pp. 257-307). San Diego: Academic Press.

LiBET, B. (1999). Do we have free will? Journal of Consciousness Studies, 6, 47-57.

LOPES, L. L., \& ODEN, G. C. (1987). Distinguishing between random and nonrandom events. Journal of Experimental Psychology: Learning, Memory, \& Cognition, 13, 392-400.

LUnG, C. T., \& Dominowski, R. L. (1985). Effects of strategy instructions and practice on nine-dot problem solving. Journal of Experimental Psychology: Learning, Memory, \& Cognition, 11, 804-811.

Machado, A. (1989). Operant conditioning of behavioral variability using a percentile reinforcement schedule. Journal of the Experimental Analysis of Behavior, 52, 155-166.

MACHADO, A. (1992). Behavioral variability and frequency-dependent selection. Journal of the Experimental Analysis of Behavior, 58, 241-263.

Machado, A. (1993). Learning variable and stereotypical sequences of responses: Some data and a new model. Behavioural Processes, 30, 103-130.

Machado, A. (1997). Increasing the variability of response sequences in pigeons by adjusting the frequency of switching between two keys. Journal of the Experimental Analysis of Behavior, 68, 1-25.

Machado, A., \& CeVIK, M. (1998). Acquisition and extinction under periodic reinforcement. Behavioural Processes, 44, 237-262.

MAIER, N. R. F. (1933). An aspect of human reasoning. British Journal of Psychology, 24, 144-155.

Maltzman, I. (1960). On the training of originality. Psychological Review, 67, 229-242.

Manabe, K., Staddon, J. E. R., \& Cleaveland, J. M. (1997). Control of vocal repertoire by reward in budgerigars (Melopsittacus undulatus). Journal of Comparative Psychology, 111, 50-62.

Manoel, E. J., \& Connolly, K. J. (1995). Variability and the development of skilled actions. International Journal of Psychophysiology, 19, 129-147.

Manoel, E. J., \& Connolly, K. J. (1997). Variability and stability in the development of skilled actions. In K. J. Connolly \& H. Forssberg (Eds.), Neurophysiologyand neuropsychologyof motor development (pp. 286-318). London: Mac Keith.

McElroy, E., \& Neuringer, A. (1990). Effects of alcohol on reinforced repetitions and reinforced variations in rats. Psychopharmacology, 102, 49-55.

Mechner, F. (1958). Sequential dependencies of the lengths of consecutive response runs. Journal of the Experimental Analysis of Behavior, 1, 229-233.

Mechner, F. (1992). Learning and practicing skilled performance. New York: Behavioral Science Applications.

Mechner, F., Hyten, C., Field, D. P., \& Madden, G. (1997). Using revealed operants to study the structure and properties of human operant behavior. Psychological Record, 47, 45-68.

MetzGer, M. A. (1994). Have subjects been shown to generate chaotic number? Commentary on Neuringer and Voss. Psychological Science, 5, 111-114.

Miller, N., \& Neuringer, A. (2000). Reinforcing variability in adolescents with autism. Journal of Applied Behavior Analysis, 33, 151-165.

Mook, D. M., Jeffrey, J., \& Neuringer, A. (1993). Spontaneously hypertensive rats (SHR) readily learn to vary but not to repeat instrumental responses. Behavioral \& Neural Biology, 59, 126-135.

Mook, D. M., \& Neuringer, A. (1994). Different effects of amphetamine on reinforced variations versus repetitions in spontaneously hypertensive rats (SHR). Physiology \& Behavior, 56, 939-944.

Morgan, L., \& Neuringer,A. (1990). Behavioral variability as a function of response topography and reinforcement contingency. Animal Learning \& Behavior, 18, 257-263.

MorRIS, C. (1987). The operant conditioning of response variability: Free-operant versus discrete-response procedures. Journal of the Experimental Analysis of Behavior, 47, 273-277.

Moxley, R. A. (1997). Skinner: From determinism to random variation. Behavior \& Philosophy, 25, 3-28.

Moxon, E. R. (1997). The tinkerer's evolving tool-box. Nature, 387, 659-662. 
Mullins, M., \& Rincover, A. (1985). Comparing autistic and normal children along the dimensions of reinforcement maximization, stimulus sampling, and responsiveness to extinction. Journal of Experimental Child Psychology, 40, 350-374.

Myerson, J., \& HAle, S. (1988). Choice in transition: A comparison of melioration and the kinetic model. Journal of the Experimental Analysis of Behavior, 49, 291-302.

Nelson, T. D. (1978). Fixed-interval matching-to-sample: Intermatching time and intermatching error runs. Journal of the Experimental Analysis of Behavior, 29, 105-113.

Neuringer, A. (1981). Self-experimentation: A call for change. Behaviorism, 9, 79-94.

Neuringer, A. (1986). Can people behave "randomly?": The role of feedback. Journal of Experimental Psychology: General, 115, 62-75.

NeUringer, A. (1991). Operant variability and repetition as functions of interresponse time. Journal of Experimental Psychology: Animal Behavior Processes, 17, 3-12.

Neuringer, A. (1992). Choosing to vary and repeat. Psychological Science, 3, 246-250.

Neuringer, A. (1993). Reinforced variation and selection. Animal Learning \& Behavior, 21, 83-91.

Neuringer, A. (2002). Reinforcement of response-sequence variability in young chicks. Unpublished manuscript.

NeUringer, A. (in press). Reinforced variability and creativity. In K. A. Lattal \& P. N. Chase (Eds.), Behavior theory and philosophy. New York: Kluwer Academic/Plenum.

Neuringer, A., Deiss, C., \& Imig, S. (2000). Comparing choices and variations in people and rats: Two teaching experiments. Behavior Research Methods, Instruments, \& Computers, 32, 407-416.

Neuringer, A., Deiss, C., \& Olson, G. (2000). Reinforced variability and operant learning. Journal of Experimental Psychology: Animal Behavior Processes, 26, 98-111.

Neuringer, A., \& Huntley, R. W. (1992). Reinforced variability in rats: Effects of gender, age and contingency. Physiology \& Behavior, 51, 145-149.

Neuringer, A., Kornell, N., \& Olufs, M. (2001). Stability and variability in extinction. Journal of Experimental Psychology: Animal Behavior Processes, 27, 79-94.

Neuringer, A., \& Voss, C. (1993). Approximating chaotic behavior. Psychological Science, 4, 113-119.

Neuringer, A., \& Voss, C. (2002). Approximations to chaotic responding depends on interresponse time. Unpublished manuscript.

NEVIN, J. A. (1967). Effects of reinforcement scheduling on simultaneous discrimination performance. Journal of the Experimental Analysis of Behavior, 10, 251-260.

Nevin, J. A. (1992). An integrative model for the study of behavioral momentum. Journal of the Experimental Analysis of Behavior, 57, 301-316.

Notterman, J. M., \& Mintz, D. E. (1965). Dynamics of response. New York: Wiley.

Page, S., \& Neuringer, A. (1985). Variability is an operant. Journal of Experimental Psychology: Animal Behavior Processes, 11, 429-452.

PETERSON, I. (1997). The jungle of randomness: A mathematical safari. New York: Wiley.

Pryor, K. W., HaAg, R., \& O'Reilly, J. (1969). The creative porpoise: Training for novel behavior. Journal of the Experimental Analysis of Behavior, 12, 653-661.

Rescorla, R. A., \& Wagner, A. R. (1972). A theory of Pavlovian conditioning: Variations in the effectiveness of reinforcement and nonreinforcement. In A. H. Black \& W. F. Prokasy (Eds.), Classical conditioning II: Current research and theory (pp. 64-99). New York: Appleton-Century-Crofts.

RhineHART, L. (1998). The dice man. Woodstock, NY: Overlook.

Roberts, S., \& Neuringer, A. (1998). Self-experimentation. In K. A. Lattal \& M. Perone (Eds.), Handbook of research methods in human operant behavior (pp. 619-655). New York: Plenum.

Roots, C. (2000). A spoonful of fish food helps the repetition go down: Reinforcing variability in Betta splendens. Unpublished undergraduate thesis, Reed College.

Ross, C., \& Neuringer, A. (2002). Reinforcement of variations and repetitions along three independent response dimensions. Behavioural Processes, 57, 199-209.
Saldana, R. L., \& Neuringer, A. (1998). Is instrumental variability abnormally high in children exhibiting ADHD and aggressive behavior? Behavioural Brain Research, 94, 51-59.

SchmidT, R. A., \& LEE, T. D. (1999). Motor control and learning: A behavioral emphasis (3rd ed.). Champaign, IL: Human Kinetics.

Schoenfeld, W. N., Harris, A. H., \& Farmer, J. (1966). Conditioning response variability. Psychological Reports, 19, 551-557.

Schwartz, B. (1980). Development of complex stereotyped behavior in pigeons. Journal of the Experimental Analysis of Behavior, 33, 153-166.

Schwartz, B. (1981). Reinforcement creates behavioral units. Behaviour Analysis Letters, 1, 33-41.

Schwartz, B. (1982). Failure to produce variability with reinforcement. Journal of the Experimental Analysis of Behavior, 37, 171-181.

Schwartz, B. (1988). The experimental synthesis of behavior: Reinforcement, behavioral stereotypy and problem solving. In G. H. Bower (Ed.), The psychology of learning and motivation (Vol. 22, pp. 93-138). New York: Academic Press.

SCRIVEN, M. (1965). An essential unpredictability in human behavior. In B. Wolman (Ed.), Scientific psychology (pp. 411-425). New York: Basic Books.

Searcy W. A., \& Yasukawa, K. (1990). Use of song repertoire in intersexual and intrasexual contexts by male red-winged blackbirds. $B e$ havioral \& Ecological Sociobiology, 27, 123-128.

SEGAL, E. F. (1972). Induction and the provenance of operants. In R. M. Gilbert \& J. R. Millenson (Eds.), Reinforcement: Behavioral analyses (pp. 1-34). New York: Academic Press.

Seligman, M. E. P. (1975). Helplessness. San Francisco: Freeman.

Senkowski, P. C., Vogel, V. A., \& Pozulp, N. C., II (1978). Differential reinforcement of lever-press durations: Effects of deprivation level and reward magnitude. Learning \& Motivation, 9, 446-465.

ShetTleworth, S. J. (1998). Cognition, evolution, and behavior. New York: Oxford University Press.

Shimp, C. P. (1967). Reinforcement of least-frequent sequences of choices. Journal of the Experimental Analysis of Behavior, 10, 57-65.

SIEGLER, R. S. (1996). Emerging minds: The process of change in children's thinking. New York: Oxford University Press.

SkINNER, B. F. (1959). Cumulative record. New York: Appleton-CenturyCrofts.

SKINNER, B. F. (1966). The phylogeny and ontogeny of behavior. Science, 153, 1205-1213.

SkINNER, B. F. (1974). About behaviorism. New York: Knopf.

SKINNER, B. F. (1981). Selection by consequences. Science, 213, 501-504.

Sмiтh, L. D. (1992). On prediction and control: B. F. Skinner and the technological ideal of science. American Psychologist, 47, 216-223.

Staddon, J. E. R., \& Simmelhag, V. L. (1971). The "superstition" experiment: A reexamination of its implications for the principles of adaptive behavior. Psychological Review, 78, 3-43.

Stein, L., Xue, B. G., \& Belluzzi, J. D. (1994). In vitro reinforcement of hippocampal bursting: A search for Skinner's atoms of behavior. Journal of the Experimental Analysis of Behavior, 61, 155-168.

Stephens, D. W., \& Krebs, J. R. (1986). Foraging theory. Princeton, NJ: Princeton University Press.

Stokes, P. D. (1995). Learned variability. Animal Learning \& Behavior, 23, 164-176.

StOKES, P. D. (2001). Variability, constraints, and creativity: Shedding light on Claude Monet. American Psychologist, 56, 355-359.

Stokes, P. D., \& BALSAM, P. [D.] (2001). An optimal period for setting sustained variability levels. Psychonomic Bulletin \& Review, 8, 177-184.

Stokes, P. D., Mechner, F., \& Balsam, P. D. (1999). Effects of different acquisition procedures on response variability. Animal Learning \& Behavior, 27, 28-41.

SupPES, P. (1984). Probabilistic metaphysics. New York: Basil Blackwell.

Sutton, R. S., \& Barto, A. G. (1998). Reinforcement learning. Cambridge, MA: MIT Press.

SutTon-Smith, B. (1975). The useless made useful: Play as variability training. School Review, 83, 197-214.

Tatham, T., Wanchisen, B., \& Hineline, P. (1993). Effects of fixed and variable ratios on human behavioral variability. Journal of the Experimental Analysis of Behavior, 59, 349-359.

Timberlake, W., \& LuCAS, G. A. (1989). Behavior systems and learning: From misbehavior to general principles. In S. B. Klein \& R. R. 
Mowrer (Eds.), Contemporary learning theories: Instrumental conditioning theory and the impact of biological constraints on learning (pp. 237-275). Hillsdale, NJ: Erlbaum.

Tremont, P. J. (1984). Variability of force and interresponse time under random interval reinforcement schedules. Behavioural Processes, 9 , 413-420.

Van der Linden, M., Beerten, A., \& Pesenti, M. (1998). Age related differences in random generation. Brain \& Cognition, 38, 1-16.

van der Meer, A. L. H., van der Weel, F. R, \& Lee, D. N. (1995). The functional significance of arm movements in neonates. Science, 267, 693-695.

Van Hest, A., van Haaren, F., \& van de Poll, N. E. (1989). Operant conditioning of response variability in male and female Wistar rats. Physiology \& Behavior, 45, 551-555.

Vollmer, T. R., Iwata, B. A., Zarcone, J. R., Smith, R. G., \& MazaLESKI, J. L. (1993). The role of attention in the treatment of attentionmaintained self-injurious behavior: Noncontingent reinforcement and differential reinforcement of other behavior. Journal of Applied Behavior Analysis, 26, 9-21.

Vollmer, T. R., Ringdahl, J. E., Roane, H. S., \& Marcus, B. A. (1997). Negative side effects of noncontingent reinforcement. Journal of Applied Behavior Analysis, 30, 161-164.

WAGENAAR, W. A. (1972). Generation of random sequences by human subjects: A critical survey of literature. Psychological Bulletin, 77, 65-72.

WARD, L. M. (2002). Dynamical cognitive science. Cambridge, MA: MIT Press.

WARD, L. M., \& WEST, R. L. (1994). On chaotic behavior. Psychological Science, 5, 232-236.
WeIss, R. L. (1964). On producing random responses. Psychological Reports, 14, 931-941.

WEISS, R. L. (1965). "Variables that influence random-generation": An alternative hypothesis. Perceptual \& Motor Skills, 20, 307-310.

WELls, M. (1999). Teaching an old rat new tricks: The effects of age on behavioral variability. Unpublished undergraduate thesis, Reed College.

Wilkes, G. (1996). Click \& Treat Training Kit [Version 1.1]. Available from www.clickandtreat.com.

Williams, B. A. (1971). Non-spatial delayed alternation by the pigeon. Journal of the Experimental Analysis of Behavior, 16, 15-21.

WILson, N. (1986). Standardsfor deviation: Tools for reinforcing variability. Unpublished undergraduate thesis, Reed College.

Winston, A. S., \& BAKER, J. E. (1985). Behavior analytic studies of creativity: A critical review. The Behavior Analyst, 8, 191-205.

Wright, A. A. (1997). Concept learning and learning strategies. Psychological Science, 8, 119-123.

Wultz, B., Sagvolden, T., Moser, E. I., \& Moser, M. B. (1990). The spontaneously hypertensive rat as an animal model of attention-deficit hyperactivity disorder: Effects of methylphenidate on exploratory behavior. Behavioral \& Neural Biology, 53, 88-102.

Young, M. E., \& WASSERMAN, E. A. (2001). Entropy and variability discrimination. Journal of Experimental Psychology: Learning, Memory, \& Cognition, 27, 278-293.

ZuRIFF, G. E. (1985). Behaviorism: A conceptual reconstruction. New York: Columbia University Press.

(Manuscript received August 14, 2001; revision accepted for publication December 19, 2001.) 